\title{
Abstracting Gradual Typing Moving Forward: Precise and Space-Efficient
}

\author{
FELIPE BAÑADOS SCHWERTER, University of British Columbia, Canada \\ ALISON M. CLARK* \\ KHURRAM A. JAFERY*, Amazon, Canada \\ RONALD GARCIA, University of British Columbia, Canada
}

\begin{abstract}
Gradual Typing (AGT) is a systematic approach to designing gradually-typed languages. Languages developed using AGT automatically satisfy the formal semantic criteria for gradual languages identified by Siek et al. Nonetheless, vanilla AGT semantics can still have important shortcomings. First, a gradual language's runtime checks should preserve the space-efficiency guarantees inherent to the underlying static and dynamic languages. To the contrary, the default operational semantics of AGT break proper tail calls. Second, a gradual language's runtime checks should enforce basic modular type-based invariants expected from the static type discipline. To the contrary, the default operational semantics of AGT may fail to enforce some invariants in

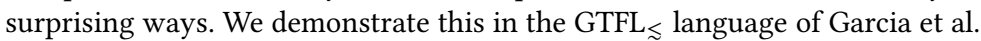

This paper addresses both problems at once by refining the theory underlying AGT's dynamic checks. Garcia et al. observe that AGT involves two abstractions of static types: one for the static semantics and one for the dynamic semantics. We recast the latter as an abstract interpretation of subtyping itself, while gradual types still abstract static types. Then we show how forward-completeness (Giacobazzi and Quintarelli) is key to supporting both space-efficient execution and reliable runtime type enforcement.
\end{abstract}

CCS Concepts: • Theory of computation $\rightarrow$ Type structures; Operational semantics; $\bullet$ Software and its engineering $\rightarrow$ General programming languages.

Additional Key Words and Phrases: gradual typing, cast calculi, abstract interpretation, subtyping

ACM Reference Format:

Felipe Bañados Schwerter, Alison M. Clark, Khurram A. Jafery, and Ronald Garcia. 2021. Abstracting Gradual Typing Moving Forward: Precise and Space-Efficient. Proc. ACM Program. Lang. 5, POPL, Article 61 (January 2021), 28 pages. https://doi.org/10.1145/3434342

\section{INTRODUCTION}

Gradual typing is an increasingly popular approach to designing programming languages that seamlessly blend dynamic and static type checking. Work in this space has produced a variety of language models, e.g., [Ina and Igarashi 2011; Sergey and Clarke 2012; Siek and Taha 2007, 2006; Tobin-Hochstadt and Felleisen 2006; Wadler and Findler 2009], evaluation criteria [Siek et al. 2015],

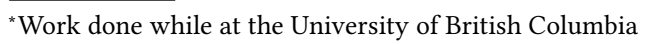

Authors' addresses: Felipe Bañados Schwerter, fbanados@cs.ubc.ca, Software Practices Lab, Department of Computer Science, University of British Columbia, 201-2366 Main Mall, Vancouver, BC, V6T1Z4, Canada; Alison M. Clark, alison. marie.clark@gmail.com; Khurram A. Jafery, khurram.jafery@gmail.com, Amazon, Canada; Ronald Garcia, rxg@cs.ubc.ca, Software Practices Lab, Department of Computer Science, University of British Columbia, 201-2366 Main Mall, Vancouver, BC, V6T1Z4, Canada.

This work is licensed under a Creative Commons Attribution 4.0 International License.

(C) 2021 Copyright held by the owner/author(s).

2475-1421/2021/1-ART61

https://doi.org/10.1145/3434342

Proc. ACM Program. Lang., Vol. 5, No. POPL, Article 61. Publication date: January 2021. 
and extensions of the concept to new contexts, e.g., [Bañados Schwerter et al. 2014; Disney and Flanagan 2011].

However, each excursion raises new questions about how language designers can produce sensible gradually-typed counterparts to their chosen static type discipline, and compare and evaluate alternative potential designs. To address these problems, Garcia et al. [2016] proposed Abstracting Gradual Typing (AGT), a methodology for developing gradually-typed extensions of pre-existing static type disciplines. Their approach systematizes the construction of static and dynamic semantics for gradual languages that by construction admit straightforward proofs of broadly accepted formal criteria for gradually typed languages [Siek et al. 2015].

Despite its metatheoretical gains, the AGT approach still requires extra work and human creativity to achieve some operational and semantic goals for a gradually-typed language. First, AGT-induced semantics do not automatically provide space-efficient runtime checking. Second, AGT's runtime checking regime sometimes requires manual tuning to ensure that in addition to Siek et al.'s criteria, desired and expected modular type-based semantic invariants are properly enforced. This paper addresses both of these concerns, but first explains them in more detail.

Space-Efficient Runtime Checking. Herman et al. [2010] showed that naïvely implementing runtime checks using wrappers can introduce space leaks and break proper tail calls. For example, consider the gradually-typed program:

$$
\begin{aligned}
\operatorname{even}(n: \operatorname{Int}): ?= & \text { if }(n=0) \text { then True else odd }(n-1) \\
\operatorname{odd}(n: \operatorname{Int}): \text { Bool } & =\text { if }(n=0) \text { then False else even }(n-1)
\end{aligned}
$$

Some terms are ascribed the unknown type?, which marks absent type information and enables runtime checking. The program looks tail-recursive, but is instrumented as follows ${ }^{1}$ :

$$
\begin{aligned}
\operatorname{even}(n: \operatorname{Int}): ?=\text { if }(n=0) \text { then }\langle ? \Leftarrow \mathrm{Bool}\rangle \text { True else }\langle ? \Leftarrow \operatorname{Bool}\rangle \text { odd }(n-1) \\
\operatorname{odd}(n: \operatorname{Int}): \text { Bool }=\text { if }(n=0) \text { then False else }\langle\text { Bool } \Leftarrow ?\rangle \text { even }(n-1)
\end{aligned}
$$

In this program, the highlighted bracket expressions denote runtime type casts. In Siek and Taha [2006] semantics, runtime casts accumulate at each recursive call:

$$
\begin{aligned}
\text { odd }(3) & \longrightarrow\langle\text { Bool } \Leftarrow ?\rangle \text { even }(2) \longrightarrow\langle\text { Bool } \Leftarrow ?\rangle\langle ? \Leftarrow \text { Bool }\rangle \text { odd }(1) \\
& \longrightarrow\langle\text { Bool } \Leftarrow ?\rangle\langle ? \Leftarrow \mathrm{Bool}\rangle\langle\text { Bool } \Leftarrow ?\rangle \text { even }(0) \\
& \longrightarrow\langle\text { Bool } \Leftarrow ?\rangle\langle ? \Leftarrow \mathrm{Bool}\rangle\langle\text { Bool } \Leftarrow ?\rangle\langle ? \Leftarrow \text { Bool }\rangle \text { True } \longrightarrow\langle\text { Bool }\rangle\langle ?\rangle \text { True } \longrightarrow \text { True }
\end{aligned}
$$

This means that programs that appear to be tail-recursive, and thus consume constant stack space, now consume linear space, simply because of how their types are checked. A variant of the same program written in continuation-passing-style demonstrates how (higher-order) casts, which cannot be resolved immediately, accumulate around values if one is not careful.

$$
\begin{aligned}
\operatorname{evenk}(n: \operatorname{Int})(k: ? \rightarrow ?): \text { Bool }=\text { if }(n=0) \text { then }(k \text { True }) \text { else oddk }(n-1) k \\
\operatorname{oddk}(n: \text { Int })(k: \text { Bool } \rightarrow \text { Bool }): \text { Bool }: \text { Bool }=\text { if }(n=0) \text { then }(k \text { False }) \text { else evenk }(n-1) k
\end{aligned}
$$

\footnotetext{
${ }^{1}$ For clarity, these examples use a type cast notation with source and destination types. Our formal semantics exhibit the same concepts, albeit using a necessarily less transparent representation.
} 
This program elaborates to the following:

$$
\begin{aligned}
& \text { evenk }(n: \text { Int })(k: ? \rightarrow ?): \text { Bool }= \\
& \quad \text { if }(n=0) \text { then }(k\langle ? \Leftarrow \text { Bool }\rangle \text { True }) \text { else oddk }(n-1)(\langle\text { Bool } \rightarrow \text { Bool } \Leftarrow ? \rightarrow ?\rangle k) \\
& \text { oddk }(n: \text { Int })(k: \text { Bool } \rightarrow \text { Bool }): \text { Bool }= \\
& \quad \text { if }(n=0) \text { then }(k \text { False }) \text { else evenk }(n-1)(\langle ? \rightarrow ? \Leftarrow \text { Bool } \rightarrow \text { Bool }\rangle k)
\end{aligned}
$$

and its evaluation also accumulates casts:

$$
\begin{aligned}
\operatorname{odd}(3) k & \longrightarrow \text { even }(2)\langle ? \rightarrow ? \Leftarrow \mathrm{Bool} \rightarrow \mathrm{Bool}\rangle k \\
& \longrightarrow \text { odd }(1)\langle\text { Bool } \rightarrow \mathrm{Bool} \Leftarrow ? \rightarrow ?\rangle\langle ? \rightarrow ? \models \mathrm{Bool} \rightarrow \mathrm{Bool}\rangle k \\
& \longrightarrow \text { even }(0)\langle ? \rightarrow ? \Leftarrow \mathrm{Bool} \rightarrow \mathrm{Bool}\rangle\langle\mathrm{Bool} \rightarrow \mathrm{Bool} \Leftarrow ? \rightarrow ?\rangle\langle ? \rightarrow ? \Leftarrow \mathrm{Bool} \rightarrow \text { Bool }\rangle k \longrightarrow \ldots
\end{aligned}
$$

The solution to this problem is to aggressively compose casts, in tail position and around values respectively, rather than allowing them to accumulate. However, doing so requires some notion of bounded-space cast-composition whose behavior is equivalent to the standard semantics. Many gradual language semantics use coercions [Henglein 1994] or threesomes [Siek and Wadler 2010] to coalesce casts as they arise. At first glance, AGT appears to immediately support space efficiency, since it provides an operator for composing checks. However, this only suffices to prevent accumulation on values. To see why, consider how space-efficient tail-recursion must proceed: ${ }^{2}$

$$
\begin{gathered}
\text { odd }(3) \longrightarrow\langle\text { Bool } \Leftarrow \text { ? }\rangle \text { even }(2) \longrightarrow\langle\text { Bool } \Leftarrow ?\rangle\langle ? \Leftarrow \text { Bool }\rangle \text { odd }(1) \longrightarrow\langle\text { Bool } \Leftarrow \text { Bool }\rangle \text { odd }(1) \\
\longrightarrow\langle\text { Bool } \Leftarrow \text { Bool }\rangle\langle\text { Bool } \Leftarrow ?\rangle \text { even }(0) \longrightarrow\langle\text { Bool } \Leftarrow \text { ? }\rangle \text { even }(0) \longrightarrow\langle\text { Bool } \Leftarrow \text { Bool }\rangle \text { True } \longrightarrow \text { True }
\end{gathered}
$$

To preserve proper tail calls the semantics must aggressively compose tail-position checks as they arise, rather than waiting until a final tail call returns a value before resolving checks up the call stack, as in the first trace. This only works if composing checks is equivalent in either direction: cast composition must be associative. Unfortunately this is not always the case: in particular, the checks introduced by Garcia et al. [2016] for $\mathrm{GTFL}_{\S}$, a gradual language with record subtyping, do not compose associatively. Some cast sequences succeed when composed in one order, but fail when composed in the other. We show a detailed example in Sec. 2.4.

Type-Based Reasoning. Prior work on gradualizing sophisticated typing disciplines like information flow security [Toro et al. 2018] or parametricity (e.g., Ahmed et al. [2017]; New et al. [2019]; Toro et al. [2019]) has demonstrated that AGT applied naïvely does not automatically enforce the modular invariants that a programmer may expect from the intended static type discipline. Unfortunately the same phenomenon arises in $\mathrm{GTFL}_{\varsigma}$, even though its type discipline-record subtyping-is comparatively simple and well-understood. ${ }^{3}$

The following scenario demonstrates the problem. Consider the following program fragment:

$$
\text { let } \mathrm{q}:[\mathrm{x}: \operatorname{lnt}]=[\mathrm{x}=5, \mathrm{y}=\text { true }] \text { in }\langle\text { body }
$$

According to standard subtype-based reasoning, the body cannot access the $y$ field of the record. Such modular reasoning is a hallmark of static typing, and programmers who mix static and dynamic typing want to reason about their code using static types where available [Tunnell Wilson et al. 2018]. Unfortunately, the following completed $\mathrm{GTFL}_{\lesssim}$ program compiles and runs successfully:

$$
\text { let } \mathrm{q}:[\mathrm{x}: \operatorname{lnt}]=[\mathrm{x}=5, \mathrm{y}=\operatorname{true}] \text { in }(\mathrm{q}:: \text { ?:: }[\mathrm{x}: \ln \mathrm{t}, \mathrm{y}: \mathrm{Bool}]) \cdot \mathrm{y}
$$

\footnotetext{
${ }^{2}$ For simplicity this example suppresses some details: see Herman et al. [2010].

${ }^{3}$ Formally speaking, information-flow and parametricity are hyperproperties: relationships among multiple runs of a program, whereas record subtyping is not.
} 
In essence, casting q to the unknown type and then back to a record type exposes the extra field that should have been hidden by q's assumed type. In other words, the language satisfies standard type safety, as AGT does by construction, but its runtime treatment of type abstractions does not strictly enforce the type abstraction properties one expects from subtyping.

Forward-completeness Is the Key. Space efficiency and type-based enforcement problems have the same source: an insufficiently-precise runtime type enforcement mechanism. Previous AGTbased systems have had to tune their runtime abstractions. Toro et al. [2018] tuned their AGT abstraction to achieve type enforcement. Guided by intuition, Toro and Tanter [2020] tuned their abstraction to achieve a space-efficient implementation. This work develops a principled approach to tuning the runtime checks in an AGT-based language, and shows that it suffices to address the two challenges above.

Our contributions are as follows:

- We revisit the foundations of AGT's dynamic checks and refine their conception. Garcia et al. [2016] conceive of them via post hoc manipulation of abstractions for tuples of types: we redefine them as a direct abstract interpretation of the subtyping relation itself. This reframing is subtle and equivalent to the original, but now techniques from the abstract interpretation literature become immediately applicable (Sec. 3).

- AGT, applied naïvely, does not support space-efficiency because composing checks out of order may not preserve behaviour. We explore this issue in the context of Garcia et al. [2016]'s $\mathrm{GTFL}_{\lesssim}$ language, and introduce bounded records and rows, a new abstraction for runtime evidence that retains precise information about gradual types and how they interact at runtime. This precise representation admits associative cast composition. Bounded rows uncover a subtle interplay between static record subtyping and gradual row types.

- As others observed, AGT-designed semantics do not automatically ensure all desired typebased reasoning principles. This problem appeared in prior work on sophisticated typing disciplines that enforce properties fundamentally about multiple runs of a program (i.e., hyperproperties). Subtyping, in contrast, is a standard unary type property, yet still requires care to enforce when gradual types are sufficiently rich. Ours is the first work to witness this phenomenon in a simple setting.

- Since AGT is a framework for designing languages, it is desirable to frame these improvements in a general form. To do so, we devise sufficient conditions for space-efficiency and precise runtime monitoring. Both properties can be achieved, while satisfying the formal criteria of Siek et al. [2015], by ensuring that the dynamic monitoring semantics is forward complete, a concept from abstract interpretation [Giacobazzi and Quintarelli 2001]. Forward completeness guarantees that an AGT semantics can be made space-efficient, and equates reasoning about the dynamic semantics to reasoning up to precision () about static subtyping.

\section{GTFL $_{\varsigma}$ : A GRADUALLY TYPED LANGUAGE}

As a concrete starting point for our investigations, this section presents the semantics of $\mathrm{GTFL}_{\lessgtr}$, a gradually-typed language with records and subtyping that also supports migration between dynamic and static type checking. Garcia et al. [2016] developed this language using the AGT methodology, and its semantics exhibits the shortcomings that this paper addresses.

We first present $\mathrm{GTFL}_{\S}$ with little reference to the AGT machinery used to construct and justify it. Furthermore, we state correctness properties here without proof, because AGT exploits calculational abstract interpretation techniques [Cousot and Cousot 1977] to intertwine the proof and definition processes, making the design “correct by construction." Sec. 3 connects GTFL $L_{\S}$ 's design to AGT, and 


\section{Syntax}

$$
x \in \text { Var, } \quad b \in \text { Bool, } \quad n \in \mathbb{Z}, \quad l \in \text { Label, } \quad \Gamma \in \text { Env }=\text { Var } \stackrel{\text { fin }}{\longrightarrow} \text { Type }
$$

$T \in$ Type $::=\operatorname{lnt} \mid$ Bool $|T \rightarrow T|[\overline{l: T}]$

$S \in$ GType $::=\quad$ | Int $\mid$ Bool $|S \rightarrow S|[\overline{l: S}] \mid[\overline{l: S}$, ?]

(static types)

$t \in$ Term $\quad:=n|b| x|\lambda x: S . t| t t|t+t|$ if $t$ then $t$ else $t|[\overline{l=t}]| t . l \mid t:: S \quad$ (terms)

\section{$\Gamma \vdash t: S \quad$ Gradual Typing}
$(S \mathrm{x}) \frac{x: S \in \Gamma}{\Gamma \vdash x: S}$
$(\mathrm{Sn}) \frac{}{\Gamma \vdash n: \operatorname{lnt}}$
(Sb) $\frac{}{\Gamma \vdash b: \text { Bool }}$
$(S$ app $) \frac{\overline{\Gamma \vdash t_{i}: S_{i}} \quad S_{2} \lesssim \widetilde{\operatorname{dom}}\left(S_{1}\right)}{\Gamma \vdash t_{1} t_{2}: \widetilde{\operatorname{cod}}\left(S_{1}\right)}$
$(S+) \frac{\overline{\Gamma \vdash t_{i}: S_{i}} \quad \overline{S_{i} \lesssim \operatorname{lnt}}}{\Gamma \vdash t_{1}+t_{2}: \operatorname{lnt}}$
(Sif) $\frac{\overline{\Gamma \vdash t_{i}: S_{i}} \quad S_{1} \lesssim \text { Bool }}{\Gamma \vdash \text { if } t_{1} \text { then } t_{2} \text { else } t_{3}: S_{2} \widetilde{V} S_{3}}$
$(S$ proj $) \frac{\Gamma \vdash t: S}{\Gamma \vdash t . l: \widetilde{p r o}(S, l)}$
$(S \lambda) \frac{\Gamma, x: S_{1} \vdash t: S_{2}}{\Gamma \vdash\left(\lambda x: S_{1} \cdot t\right): S_{1} \rightarrow S_{2}}$
$(S::) \frac{\Gamma \vdash t: S \quad S \lesssim S_{1}}{\Gamma \vdash\left(t:: S_{1}\right): S_{1}}$
$\left(\right.$ Srec) $\frac{\overline{\Gamma \vdash t_{i}: S_{i}}}{\Gamma \vdash\left[\overline{l_{i}=t_{i}}\right]:\left[\overline{l_{i}: S_{i}}\right]}$

\section{Helper Functions}

$\widetilde{d o m}:$ GType $\rightarrow$ GType

$\widetilde{\operatorname{dom}}\left(S_{1} \rightarrow S_{2}\right)=S_{1}$

$\widetilde{\operatorname{dom}}(?)=$ ?

$\widetilde{\operatorname{dom}}(S)$ undefined otherwise

$$
\begin{aligned}
& \widetilde{\operatorname{cod}}: \text { GType } \rightarrow \text { GType } \\
& \operatorname{cod}\left(S_{1} \rightarrow S_{2}\right)=S_{2} \\
& \frac{\operatorname{cod}}{\operatorname{cod}}(?)=?
\end{aligned}
$$

\begin{tabular}{|c|c|c|c|c|c|c|}
\hline \multirow[b]{3}{*}{$S_{21} \lesssim S_{11}$} & \multirow{2}{*}{ 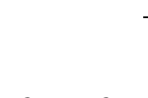 } & & & & \multirow{3}{*}{ Bool $\lesssim$ Bool } & \\
\hline & & $? \lesssim S$ & $S \lesssim ?$ & $\ln t \lesssim \operatorname{Int}$ & & \\
\hline & $S_{12} \lesssim S_{22}$ & \multicolumn{3}{|c|}{$\overline{S_{i 1} \lesssim S_{i 2}}$} & & $\overline{S_{i 1} \lesssim S_{i 2}}$ \\
\hline$S_{11}$ & $S_{21} \rightarrow S_{22}$ & & $\overline{l_{j}:}$ & {$\left[\overline{l_{i}: S_{i 2}}, *\right]$} & {$\left[\overline{l_{i}: S_{i 1}}, \overline{l_{j}: S}\right.$} & , ?] $\lesssim\left[\overline{l_{i}: S_{i 2}}, \overline{l_{k}: S_{k}}, *\right]$ \\
\hline
\end{tabular}

\section{$S \lesssim S \quad$ Consistent Subtyping}

$S \sqsubseteq S \quad$ Precision

$$
\begin{gathered}
\frac{S_{11} \sqsubseteq S_{21} \quad S_{12} \sqsubseteq S_{22}}{S_{11} \rightarrow S_{12} \sqsubseteq S_{21} \rightarrow S_{22}} \frac{\overline{S_{1 i} \sqsubseteq S_{2 i}}}{\left[\overline{l_{i}: S_{1 i}}\right] \sqsubseteq\left[\overline{l_{i}: S_{2 i}}\right]} \frac{\overline{S_{1 i} \sqsubseteq S_{2 i}}}{\left[\overline{l_{i}: S_{1 i}}, \overline{l_{j}: S_{1 j}}, *\right] \sqsubseteq\left[\overline{l_{i}: S_{2 i}}, ?\right]} \\
\frac{S \sqsubseteq ?}{\text { Int } \sqsubseteq \text { Int }} \frac{\text { Bool } \sqsubseteq \text { Bool }}{}
\end{gathered}
$$

Fig. 1. GTFL $L_{\lesssim}$ Static Semantics

briefly connects the correctness properties to proofs. Ultimately our improvements are presented in terms of AGT to ensure that they generalize across AGT-based languages.

\subsection{Syntax and Typing}

Fig. 1 presents the $\mathrm{GTFL}_{\lesssim}$ syntax and type system. Its terms are typical: numbers, booleans, functions, records, and type ascriptions. All of the novelty lies in its type structure, where common static types-atomics, functions, and records-are augmented with two gradual type constructs that denote imprecise type information. The now-standard unknown type ? denotes the complete omission of type information [Siek and Taha 2006]. The gradual row type [ $\overline{\ell: S}$, ?], on the other 
hand, represents a record type with incomplete field information. It surely constrains the list $\bar{l}$ of fields with corresponding (gradual) types $\bar{S}$, but the gradual row designator ? denotes the possibility of additional statically unknown fields. ${ }^{4}$ Gradual rows are somewhat analogous to polymorphic rows [Rémy 1989], except that their presence induces dynamic checks. As such, a gradual row type is only partially static with respect to record fields.

As is now standard for gradual typing, the concept of "imprecise type information" is formalized using the precision judgment $S_{1} \sqsubseteq S_{2}$, which says that $S_{1}$ is less imprecise than $S_{2}$. Static types are the least elements of this partial order, while the greatest element is the unknown type?

The rules for the typing judgment $\Gamma \vdash t: S$ are structured in the style of Garcia and Cimini [2015]: each typing judgment in the premise of a rule has an arbitrary type metavariable $S_{i}$, but these premise types are constrained by side conditions. The result type in the conclusion of a rule is either a particular type or a (partial) function of its premise types. For example, the two premises of the (Sapp) rule have types $S_{1}$ and $S_{2}$ respectively; these premise types are constrained by the consistent subtyping side condition $S_{2} \lesssim \widetilde{\operatorname{dom}}\left(S_{1}\right)$; and the result type (Sapp) is the gradual codomain $\widetilde{\operatorname{cod}}\left(S_{1}\right)$ of the operator type. This structure keeps the typing rules syntax-directed, while subsuming the type system for the corresponding statically typed language $\mathrm{STFL}_{<}$: (introduced by Garcia et al. [2016]). Consider, for instance, how $\operatorname{cod}\left(S_{1}\right)$ extends the idea of "the codomain of a function type". For function types $S_{1} \rightarrow S_{2}$, its behaviour is as expected, but for the unknown type ?, the codomain is completely unknown because the operator's type is completely unknown: the operator may have a function type, but we do not know for sure. We formalize this behaviour as a correctness criterion.

Definition 2.1 (Candidate Codomain).

(1) A plausible function type is a gradual type $S$ such that $T_{1} \rightarrow T_{2} \sqsubseteq S$ for some $T_{1}, T_{2} \in$ Type;

(2) A gradual type $S^{\prime}$ is a candidate codomain of a plausible function type $S$ if $T_{1} \rightarrow T_{2} \sqsubseteq S$ implies $T_{2} \sqsubseteq S^{\prime}$, for all $T_{1}, T_{2} \in$ Type.

Proposition 2.2 (Codomain Correctness). A gradual type $S$ has a candidate codomain if and only if it has a least (with respect to $\sqsubseteq$ ) candidate codomain, denoted $\widetilde{c o d}(S)$.

Prop. 2.2 implicitly defines $\widetilde{c o d}$ as a partial function on GType, which is defined for exactly the plausible function types. The notion of "candidate codomain" can be interpreted as a soundness property of cod: it broadly approximates the idea of "codomain" even in the face of imprecision. The proposition can then be interpreted as an optimality property: there is a "best" candidate codomain, if any, so $\widetilde{c o d}$ never loses precision needlessly. Analogous correctness criteria apply to the other type operators, completely characterizing the gradual versions in terms of their static counterparts.

The consistent subtyping relation $\lesssim$ [Siek and Taha 2007] extends static subtyping <: to optimistically account for imprecision in gradual types. In essence, $S_{1} \lesssim S_{2}$ means that it is plausible that $S_{1}$ is a subtype of $S_{2}$, when the imprecision of gradual types is taken into account. For instance, ? is both a consistent supertype and consistent subtype of each gradual type $S$, because it could represent any static type whatsoever, including $S$ itself. As above, we formally relate consistent subtyping to static subtyping ${ }^{5}$.

Proposition 2.3. $S_{1} \lesssim S_{2}$ if and only if $T_{1}<: T_{2}$ for some $T_{1} \sqsubseteq S_{1}$ and $T_{2} \sqsubseteq S_{2}$.

One key benefit to defining gradual operators and relations using static counterparts and gradual type precision is that the resulting language naturally satisfies static criteria for gradual typing set

\footnotetext{
${ }^{4}$ Throughout we use overlines to denote zero or more repetitions, +-annotated overlines to denote one or more repetitions, and $[\overline{l: S}, *]$ to simultaneously denote both traditional record types $[\overline{l: S}]$ and gradual row types $[\overline{l: S}$, ?]

${ }^{5}$ For $\mathrm{STFL}_{<:}$, static subtyping $<$: is standard relation induced by width and depth record subtyping [Pierce 2002]
} 
forth by Siek et al. [2015]. First, the $\mathrm{STFL}_{<:}$type system can be recovered from that of $\mathrm{GTFL}_{\varsigma}$ by simply restricting source programs to only mention static types $T$. Doing so: restricts the $\widetilde{d o m}, \widetilde{c o d}$, and $\overline{p r o j}$ partial functions to simple arrow type and record type destructors; restricts the consistent subtyping relation $\lesssim$ to a typical definition of static subtyping $<: ;$ and restricts the $\widetilde{V}$ partial function to the subtype join partial function $\ddot{v}$, which yields the least upper bound of two static types (if there is one) according to static subtyping <:. Thus, by construction, $\mathrm{GTFL}_{\lessgtr}$ conservatively extends the static language. Furthermore, the static gradual guarantee, which ensures that increasing the precision of a program's types cannot fix extant type errors, follows from the correctness criteria for each operator and relation, each of which monotonically preserves this property, yielding a direct compositional proof.

Though the syntax of gradual rows is simple, its implications for the language semantics are nontrivial. Gradual rows expose a subtle interplay between gradual type precision and static width subtyping that does not arise in most gradual type systems developed to date. Consider $[x:$ Int, $y:$ Bool $] \lesssim[x:$ Int, ?]: This judgement is justified by two static subtypings: $[x:$ Int, $y:$ Bool $]<:[x: \operatorname{lnt}]$ and $[x: \operatorname{Int}, y:$ Bool $]<:[x:$ Int, $y:$ Bool $]$. If this instance of consistent subtyping is viewed as a form of coercion, then it indicates two different behaviours: in the first the $y$ field is obscured via static subtyping. In the second, the y field is obscured via precision: not by static subtyping, but by gradual typing. The static and runtime semantics must reckon with these two different explanations simultaneously, and all outcomes must be consistent with one, the other, or both. This happens in no other language featuring consistent subtyping, and can be improved upon with a more precise abstraction. The abstraction of Garcia et al. [2016] sometimes loses information at runtime that was obscured due to loss of precision (See next paragraph). By contrast, the BRR abstraction introduced in Sec. 5 preserves all obscured information at runtime, but hides some of it from the programmer: the abstraction always reflects instances of static subtyping.

An Example. The following (somewhat contrived) example program demonstrates some of the features and intended capabilities of $\mathrm{GTFL}_{\lesssim}$, especially the semantics of gradual rows. For succinctness, we assume let binding, which can be easily added to the language.

$$
\begin{gathered}
\text { let sum }=\lambda(\text { has } M: \text { Bool }) \cdot \lambda(x:[f: \text { Int, } ?]) \\
\text { if hasM then } x \cdot f+x \cdot m \text { else } x \cdot f+x \cdot q \\
\text { in }(\text { sum true }[f=6, m=2])+(\text { sum false }[f=6, q=2])
\end{gathered}
$$

The sum function takes a record $\mathrm{f}$, and a Boolean value has $\mathrm{M}$, and uses has $\mathrm{M}$ to determine which field to add to x.f. The gradual row type ascribed to $x$ ensures statically that the record argument contains an $f$ field of type Int, but makes no commitment regarding what other fields may be present. Thus the body of the function type checks despite referring to x.m and x.q.

The program successfully calculates the sum for two different records that have different fields. The has $\mathrm{M}$ argument indicates which branch of execution is the right one. This small program demonstrates the possibility of at least partially statically checking the program, while deferring checks for extra fields to runtime. We can extrapolate from this program to a larger application that fruitfully exploits field dynamism, while statically checking stable record components.

This language design makes the possibility of dynamic checking evident in the types. The gradual type [ $f$ : Int, ?] indicates that the record may have more fields, which might be used by the program.

Furthermore, the type structure of a program can be exploited to control the amount and scope of necessary dynamic checking. For instance, if the branches of the conditional made repeated use of extra fields, then each access would require a runtime check. However, we can ensure that these are checked statically (and centralize the type assumptions) using a type ascription. For example, 
we can replace the consequent branch $x . f+x \cdot m$ with

$$
\text { let } y=(x::[f: \operatorname{lnt}, m: \operatorname{lnt}]) \text { in } y \cdot f+y \cdot m
$$

This change would move the initial field check (for x.m) and field type check (that its type is Int) to the ascription: the body of the consequent let would be fully statically checked. This use of ascription is similar to downcasting in object-oriented languages, but is justified by the presence of the imprecise gradual row in the type of sum's argument.

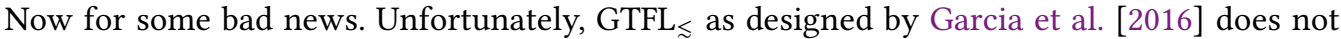
enforce its type abstractions to the extent that one might expect. Sound gradual type regimes typically have the property that precise type information is persistent . For instance, if we replace the record $[f=6, m=2]$ in the example with $([f=6, m=2]::[f: \operatorname{lnt}])$, then one would expect the program to type check, but fail at runtime. In this way, the language would guarantee that the $m$ field's very existence is encapsulated: no client would be able to violate that guarantee.

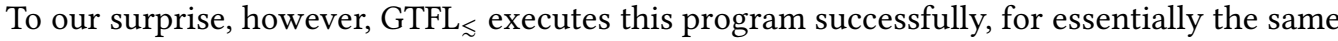
reason that the errant example from Sec. 1 succeeds. We consider this a flaw in the $\mathrm{GTFL}_{\lessgtr}$ semantics: type abstractions should be respected. Bear in mind that a language designer may desire a gradual language that treats all gradual record types as gradual rows, thereby baking downcasting into the

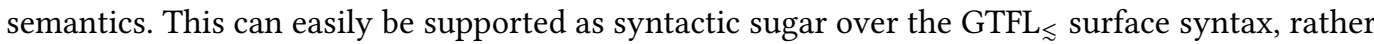
than as an accident of AGT-induced semantics. In short, the semantics of an AGT-induced language should precisely enforce types. In the following, we diagnose this failure of type abstraction and show how to properly ensure this with a refinement to the AGT framework.

\subsection{Runtime Language}

Since a gradual language defers some checks to runtime, its dynamic semantics must account for

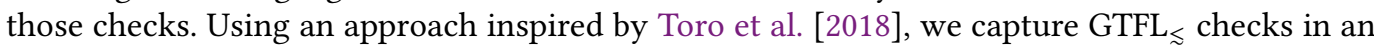
internal language that decorates programs with runtime type information.

2.2.1 Syntax and Static Semantics. Fig. 2 presents the syntax and type system of the GTFL $\mathrm{Runtime}_{\lessgtr}$ Language (RL). RL mirrors $\mathrm{GTFL}_{\S}$, but for a few differences. First, runtime terms have no source typing information: since RL programs are constructed by type-directed translation from $\mathrm{GTFL}_{\varsigma}$ (Sec. 2.3), we can presume the existence of a corresponding typing derivation, and we need not reconstruct one by analyzing RL terms. Omitting static types also lets us formally distinguish type information that is used for static checking from information that is used for runtime enforcement

Second, many RL terms, in particular the subterms of elimination forms and ascriptions, are decorated with evidence objects $\varepsilon$, which summarize runtime consistent subtyping judgments. Recall that consistent subtyping denotes the plausibility that a subtyping relationship holds between two gradual types. Evidence objects reify that plausibility, and evolve as part of runtime type enforcement. We describe this in more detail below.

The typing judgment for runtime terms is unusual in that it classifies a runtime term e with respect to a source typing judgment $\Gamma \vdash t: S$. The judgment $\mathbf{e} \vdash \Gamma \vdash t: S$ says that the runtime term e represents a source gradual typing derivation $\Gamma \vdash t: S$, where $t$ is a source-language $\mathrm{GTFL}_{\lesssim}$ term. The motivation for this structure is that in a gradually-typed language, type enforcement is not necessarily completed at type-checking time. Some type enforcement may be deferred to runtime, and this enforcement is construed as an attempt to complete (or refute) the type safety argument at runtime. Thus one can think of an RL term's execution as playing out type progress and preservation, which may ultimately fail and justifiably signal a runtime type error. In short, RL terms represent the computationally relevant residual of a $\mathrm{GTFL}_{\lesssim}$ typing derivation. 


\section{Syntax}

$$
\begin{aligned}
& \varepsilon \in \mathrm{Ev} \quad=\quad\left\{\left\langle S_{1}, S_{2}\right\rangle \mid \vdash\left\langle S_{1}, S_{2}\right\rangle \text { wf }\right\} \quad \text { (evidence objects) } \\
& \mathbf{e} \in \text { RTerm }::=\mathbf{n}|\mathbf{b}| \mathbf{x}|\lambda \mathbf{x . e}| \varepsilon \mathbf{e} \varepsilon \mathbf{e}|\varepsilon \mathbf{e}+\varepsilon \mathbf{e}| \\
& \mathbf{u} \in \text { RawValue }::=\mathbf{n}|\mathbf{b}| \mathbf{x}|\lambda \mathbf{x} . \mathbf{e}|[\overline{\mathbf{l}=\mathbf{v}}] \quad \text { (raw values) } \\
& \mathbf{v} \in \text { Value }::=\mathbf{u} \mid \varepsilon \mathbf{u} \quad \text { (values) }
\end{aligned}
$$

e $\rightarrow \Gamma \vdash t: S \quad$ Runtime Typing

$$
(\mathrm{Sn}) \frac{\mathbf{n} \vdash \Gamma+n: \operatorname{lnt}}{\mathrm{n}}
$$

$(S \mathrm{~b}) \frac{}{\mathbf{b} \triangleright \Gamma+b: \text { Bool }}$

$(S+) \frac{\overline{\mathbf{e}_{i} \vdash \Gamma \vdash t_{i}: S_{i}} \quad \overline{\varepsilon_{i} \vdash S_{i} \lesssim \operatorname{Int}}}{\left(\varepsilon_{1} \mathbf{e}_{1}+\varepsilon_{2} \mathbf{e}_{2}\right) \triangleright \Gamma \vdash t_{1}+t_{2}: \operatorname{lnt}} \quad(S a p p) \frac{\overline{\mathbf{e}_{i} \vdash \Gamma \vdash t_{i}: S_{i}} \quad \varepsilon_{1} \vdash S_{1} \lesssim S_{1}^{\prime} \rightarrow S_{2}^{\prime} \quad \varepsilon_{2} \vdash S_{2} \lesssim S_{1}^{\prime}}{\left(\varepsilon_{1} \mathbf{e}_{1} \varepsilon_{2} \mathbf{e}_{2}\right) \vee \Gamma \vdash t_{1} t_{2}: S_{2}^{\prime}}$

$($ Sif $) \frac{\overline{\mathbf{e}_{i} \vdash \Gamma \vdash t_{i}: S_{i}} \quad \varepsilon_{1} \vdash S_{1} \lesssim \text { Bool } \quad \varepsilon_{2} \vdash S_{2} \lesssim S_{2} \widetilde{\nabla} S_{3} \quad \varepsilon_{3} \vdash S_{3} \lesssim S_{2} \widetilde{\nabla} S_{3}}{\left(\text { if } \varepsilon_{1} \mathbf{e}_{1} \text { then } \varepsilon_{2} \mathbf{e}_{2} \text { else } \varepsilon_{3} \mathbf{e}_{3}\right) \triangleright \Gamma \vdash \text { if } t_{1} \text { then } t_{2} \text { else } t_{3}: S_{2} \widetilde{\nabla} S_{3}}$

$$
(S \text { proj }) \frac{\mathrm{e} \vee \Gamma \vdash t: S \quad \varepsilon \vdash S \lesssim\left[l: S^{\prime}\right]}{\varepsilon \mathbf{e} . l \vee \Gamma \vdash t . l: S^{\prime}} \quad(S \lambda) \frac{\mathrm{e} \vee \Gamma, x: S_{1} \vdash t: S_{2}}{(\lambda x . \mathbf{e}) \vee \Gamma \vdash\left(\lambda x: S_{1} . t\right): S_{1} \rightarrow S_{2}}
$$

$(S::) \frac{\mathbf{e} \triangleright \Gamma \vdash t: S \quad \varepsilon \vdash S \lesssim S^{\prime}}{\varepsilon \mathbf{e} \vdash \Gamma \vdash\left(t:: S^{\prime}\right): S^{\prime}} \quad(S \mathrm{x}) \frac{x: S^{\prime} \in \Gamma}{\mathrm{x} \triangleright \Gamma \vdash x: S^{\prime}} \quad(S \mathrm{rec}) \frac{\overline{\mathbf{e}_{i} \vdash \Gamma \vdash t_{i}: S_{i}}}{\left[\overline{\bar{l}_{i}=\mathbf{e}_{i}}\right] \triangleright \Gamma \vdash\left[\overline{l_{i}=t_{i}}\right]:\left[\overline{l_{i}: S_{i}}\right]}$

\begin{tabular}{|l|l|l}
$\varepsilon \vdash S_{1} \lesssim S_{2}$ & Evidence for Consistent Subtyping
\end{tabular}

$$
\frac{\vdash\left\langle S_{1}^{\prime}, S_{2}^{\prime}\right\rangle \text { wf } \quad S_{1}^{\prime} \sqsubseteq S_{1} \quad S_{2}^{\prime} \sqsubseteq S_{2}}{\left\langle S_{1}^{\prime}, S_{2}^{\prime}\right\rangle \vdash S_{1} \lesssim S_{2}}
$$

$r \varepsilon \mathbf{w f}$ Well-formed Evidence

$$
\begin{aligned}
& \frac{S \in\{\text { Int, Bool, ? }\}}{\vdash\langle S, S\rangle \text { wf }} \\
& \frac{\vdash\left\langle S_{21}, S_{11}\right\rangle \mathrm{wf} \quad \vdash\left\langle S_{12}, S_{22}\right\rangle \mathrm{wf}}{\vdash\left\langle S_{11} \rightarrow S_{12}, S_{21} \rightarrow S_{22}\right\rangle \mathrm{wf}} \\
& \frac{\overline{\vdash\left\langle S_{i 1}, S_{i 2}\right\rangle \mathbf{w f}} \quad\left\langle *_{1}, *_{2}\right\rangle \neq\langle\cdot, ?\rangle}{\vdash\left\langle\left[\overline{l_{i}: S_{i 1}}, *_{1}\right],\left[\overline{l_{i}: S_{i 2}}, *_{2}\right]\right\rangle \mathbf{w f}} \\
& \frac{\overline{\vdash\left\langle S_{i 1}, S_{i 2}\right\rangle \mathrm{wf}}}{\vdash\left\langle\left[\overline{\bar{l}_{i}: S_{i 1}}, \overline{l_{j}: S_{j}}, *_{1}\right],\left[\overline{l_{i}: S_{i 2}}, *_{2}\right]\right\rangle \text { wf }}
\end{aligned}
$$

Fig. 2. GTFL ${ }_{\lesssim}$ : Runtime Language Static Semantics

Naturally, the structure of RL typing rules closely mirror those of $\mathrm{GTFL}_{\varsigma}$, but differ on a few details. First, the (Sx) and (S::) rules show how missing source type information appears only as part of the corresponding source derivation, and not as part of the term. Gradual types highlighted in grey are artifacts of source type-checking that cannot be recovered by examining the runtime term itself. We exploit this information only to establish the type safety of RL programs: the runtime never needs to reconstruct a typing derivation from a naked RL term. Second, each instance of consistent subtyping from $\mathrm{GTFL}_{\lessgtr}$ is now decorated with an evidence object that supports the judgment. For instance, the RL term $\varepsilon \mathbf{e}$ corresponds via typing directly to an ascription expression $t:: S_{0}$. The evidence judgment $\varepsilon \vdash S \lesssim S_{0}$ says that $\varepsilon$ confirms that $S \lesssim S_{0}$ is plausible. 
The (Sif) and (Sapp) rules consider evidence for extra consistent subtyping judgments that were only implicitly required by the $\mathrm{GTFL}_{\lessgtr}$ rules. For instance, (Sapp) demands that $S_{1} \lesssim \widetilde{\operatorname{dom}}\left(S_{1}\right) \rightarrow \widetilde{\operatorname{cod}}\left(S_{1}\right)$. This extra constraint is implied by the GTFL $\mathrm{GL}_{\lessgtr}$ requirement that $\widetilde{\operatorname{dom}}\left(S_{1}\right)$ and $\operatorname{cod}\left(S_{1}\right)$, an argument and a result type, respectively, are well defined. The elaboration rule, and the corresponding RL term, make this implicit constraint explicit so as to enforce type structure at runtime. Similarly, the (Sif) type $S_{2} \widetilde{V} S_{3}$ imposes implicit consistent subtyping constraints on each branch of the conditional.

In RL, an evidence object is a pair of gradual types that are tightly related to one another, and also related to the consistent subtyping judgment that they support. The well-formedness judgment $\vdash \varepsilon \mathbf{w f}$ imposes several invariants on evidence.

Proposition 2.4. The judgment $\vdash\left\langle S_{1}, S_{2}\right\rangle$ wf is equivalent to the combination of the following criteria:

(1) $S_{1} \lesssim S_{2}$, i.e. $T_{1}<: T_{2}$ for some $T_{1} \sqsubseteq S_{1}$ and $T_{2} \sqsubseteq S_{2}$.

(2) Suppose $S_{1}^{\prime}, S_{2}^{\prime} \in$ GType and whenever $T_{1}<: T_{2}, T_{1} \sqsubseteq S_{1}$, and $T_{2} \sqsubseteq S_{2}$, we also have $T_{1} \sqsubseteq S_{1}^{\prime}$ and $T_{2} \sqsubseteq S_{2}^{\prime}$. Then $S_{1} \sqsubseteq S_{1}^{\prime}$ and $S_{2} \sqsubseteq S_{2}^{\prime}$.

In short, an evidence object is the least (with respect to $\sqsubseteq$ ) pair of gradual types that subsumes some set of static subtype instances $T_{1}<: T_{2}$. In this sense they are minimal sound representatives of consistent subtyping. For instance, Int $\lesssim$ ?, but $\langle$ Int, ?〉 is not well-formed evidence, because Int $<$ : Int is the only pair of static types that justifies that particular consistent subtyping instance. The well-formed evidence object $\langle$ Int, Int $\rangle$ captures the same information. From the definition of well-formed evidence, it follows immediately that if $\varepsilon \vdash S_{1} \lesssim S_{2}$ for any $\varepsilon$, then indeed $S_{1} \lesssim S_{2}$ holds in general. Evidence ensures that Prop. 2.4 is maintained during evaluation.

2.2.2 Dynamic Semantics. Fig. 3 presents a reduction semantics for RL. The notions of reduction augment standard reduction steps with operations that manipulate evidence. We first consider these reductions at a high-level, and then delve into the role of evidence in these reductions. Addition ignores its associated evidence and behaves as usual. The intuition behind this is that the evidence is now superfluous: $\mathbf{n}_{1}$ and $\mathbf{n}_{2}$ evidently have type Int because they are integers. Similarly, conditionals ignore the evidence associated with the predicate, because it is evidently a Boolean value. The chosen branch's evidence is propagated as-is to enforce its type invariants. Record projection selects the relevant field of a record, but also applies the iproj operator to the evidence that is associated to the projection operator, in order to extract evidence that is relevant to the projected value. Function application is the most complex rule. Using the idom and icod operators, it extracts evidence associated with the domain and codomain of the function subterm, composes the domain evidence with the argument evidence, and then associates the codomain evidence with the eventual result of the call. If composition fails, then the entire application fails. ${ }^{6}$

To understand the above behaviours, it helps to think of the operators like iproj as runtime representations of inversion principles on consistent subtyping. For instance, given the inductive definition of consistent subtyping in Fig. 1, we have the following inversion lemma.

Proposition 2.5. If $l_{k} \in\left\{\overline{l_{j}}\right\}$ and $\left[\overline{l_{i}: S_{i}}\right] \lesssim\left[\overline{l_{j}: S_{j}^{\prime}}\right]$ then $S_{k} \lesssim S_{k}^{\prime}$.

The iproj operator reifies this inversion principle (and those for gradual rows) at runtime: If $\varepsilon \vdash\left[\overline{l_{i}: S_{i}}\right] \lesssim\left[\overline{l_{j}: S_{j}^{\prime}}\right]$ then $\operatorname{iproj}\left(\varepsilon, l_{k}\right) \vdash S_{k} \lesssim S_{k}^{\prime}$. So just as inversion principles are used to prove static type safety, evidence inversion operators are used to enforce type invariants at runtime.

\footnotetext{
${ }^{6}$ Appendix B in the accompanying technical report [Bañados Schwerter et al. 2020] directly defines composition; we provide a succinct indirect definition in Sec. 3 using some additional concepts.
} 


\section{Syntax}

$$
\begin{array}{rlll}
E \in \text { ECtxt } & ::= & \square|E[[\overline{l=\mathbf{v}}, l=\square, \overline{l=\mathbf{e}}]]| E[F[\varepsilon \square] & \text { (evaluation contexts) } \\
F \in \text { EvFrame } & ::= & \square|\square+\varepsilon \mathbf{e}| \varepsilon \mathbf{u}+\square|\square \varepsilon \mathbf{e}| \varepsilon \mathbf{u}|\square| l & \\
& \mid & \text { if } \square \text { then } \varepsilon \mathbf{e} \text { else } \varepsilon \mathbf{e} & \text { (evidence frame) }
\end{array}
$$

\section{$\mathrm{e} \sim \mathrm{e}, \mathrm{e} \sim$ error $\quad$ Notions of Reduction}

$$
\begin{aligned}
\varepsilon_{1} \mathbf{n}_{1}+\varepsilon_{2} \mathbf{n}_{2} & \leadsto \mathbf{n}_{3} \\
\varepsilon_{1}(\lambda \mathbf{x} . \mathbf{e}) \varepsilon_{2} \mathbf{u} & \sim \begin{cases}\left(i \operatorname{cod}\left(\varepsilon_{1}\right)\right)\left(\left[\left(\varepsilon_{3} \mathbf{u}\right) / \mathbf{x}\right] \mathbf{e}\right) \\
\text { error } & \text { if } \varepsilon_{3} \text { not defined }\end{cases}
\end{aligned}
$$

if $\varepsilon_{1}$ true then $\varepsilon_{2} \mathbf{e}_{2}$ else $\varepsilon_{3} \mathbf{e}_{3} \leadsto \varepsilon_{2} \mathbf{e}_{2}$

if $\varepsilon_{1}$ false then $\varepsilon_{2} \mathbf{e}_{2}$ else $\varepsilon_{3} \mathbf{e}_{3} \leadsto \varepsilon_{3} \mathbf{e}_{3}$

$$
\varepsilon\left[\overline{l_{i}=\mathbf{v}_{i}}\right] . l_{j} \leadsto \operatorname{iproj}\left(\varepsilon, l_{j}\right) \mathbf{v}_{j}
$$

\section{$\mathrm{e} \longrightarrow \mathrm{e}, \mathrm{e} \longrightarrow$ error $\quad$ Contextual Reduction}

$\frac{\mathbf{e} \leadsto \mathbf{e}^{\prime}}{E[\mathbf{e}] \longrightarrow E\left[\mathbf{e}^{\prime}\right]} \frac{\mathbf{e} \leadsto \text { error }}{E[\mathbf{e}] \longrightarrow \text { error }} \frac{\varepsilon_{2} \stackrel{\circ}{9} \varepsilon_{1} \text { not defined }}{E\left[F\left[\varepsilon_{1} \varepsilon_{2} \mathbf{u}\right]\right] \longrightarrow \text { error }} \frac{}{E\left[F\left[\varepsilon_{1} \varepsilon_{2} \mathbf{u}\right]\right] \longrightarrow E\left[F\left[\left(\varepsilon_{2} \stackrel{\circ}{9} \varepsilon_{1}\right) \mathbf{u}\right]\right]}$

\section{Helper Functions}

$$
\begin{aligned}
& \text { FunEv }=\left\{\varepsilon \in \mathrm{Ev} \mid \varepsilon \vdash S_{11} \rightarrow S_{12} \lesssim S_{21} \rightarrow S_{22}\right\} \\
& \text { IProjDomain }=\left\{\langle\varepsilon, l\rangle \in \text { Ev } \times \text { Label } \mid \varepsilon \vdash\left[l: S, \overline{l_{i}: S_{i}}, *_{1}\right] \lesssim\left[l: S^{\prime}, \overline{l_{j}: S_{j}}, *_{2}\right]\right\} \\
& \begin{array}{ll}
\text { idom }: \text { FunEv } \rightarrow \text { Ev } & i \operatorname{cod}: \text { FunEv } \rightarrow \text { Ev } \\
i \operatorname{dom}\left(S_{1}, S_{2}\right)=\left\langle\widehat{\operatorname{dom}}\left(S_{2}\right), \widetilde{\operatorname{dom}}\left(S_{1}\right)\right\rangle & i \operatorname{cod}\left(S_{1}, S_{2}\right)=\left\langle\widetilde{\operatorname{cod}}\left(S_{1}\right), \widetilde{\operatorname{cod}}\left(S_{2}\right)\right\rangle
\end{array} \\
& \text { iproj : IProjDomain } \rightarrow \text { Ev } \\
& \operatorname{iproj}\left(\left\langle S_{1}, S_{2}\right\rangle, l\right)=\left\langle\widetilde{\operatorname{proj}}\left(S_{1}, l\right), \widetilde{\operatorname{proj}}\left(S_{2}, l\right)\right\rangle
\end{aligned}
$$

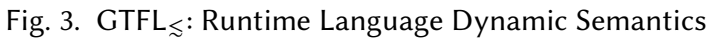

The three inversion operators are total functions over appropriate subdomains of evidence, which properly reflect the kind of runtime evidence that they are used to manipulate during reduction. As such, inversion never fails if it is encountered.

While evidence inversion operations are used to simluate inversion principles, evidence composition $\varepsilon_{1} \stackrel{\circ}{9} \varepsilon_{2}$ is used to monitor transitivity of consistent subtyping. For this reason, this composition operation is called consistent transitivity [Garcia et al. 2016]. The defining correctness criteria for consistent transitivity are as follows.

Definition 2.6. Suppose $\varepsilon_{1}=\left\langle S_{1}^{\prime}, S_{21}^{\prime}\right\rangle \vdash S_{1} \lesssim S_{2}$ and $\varepsilon_{2}=\left\langle S_{22}^{\prime}, S_{3}^{\prime}\right\rangle \vdash S_{2} \lesssim S_{3}$.

(1) Call $\left(\varepsilon_{1}, \varepsilon_{2}\right)$ plausibly transisitive if $T_{1} \sqsubseteq S_{1}^{\prime}$ and $S_{21}^{\prime} \sqsupseteq T_{2} \sqsubseteq S_{22}^{\prime}$, and $T_{3} \sqsubseteq S_{3}^{\prime}$ hold for some triple $T_{1}<: T_{2}<: T_{3}$

(2) call $\varepsilon^{\prime}=\left\langle S_{1}^{\prime \prime}, S_{3}^{\prime \prime}\right\rangle$ a candidate for transitivity of $\left(\varepsilon_{1}, \varepsilon_{2}\right)$ if $\left(\varepsilon_{1}, \varepsilon_{2}\right)$ is plausibly transisitive and every triple $T_{1}<: T_{2}<: T_{3}$ such that $T_{1} \sqsubseteq S_{1}^{\prime}$ and $S_{21}^{\prime} \sqsupseteq T_{2} \sqsubseteq S_{22}^{\prime}$, and $T_{3} \sqsubseteq S_{3}^{\prime}$ implies $T_{1} \sqsubseteq S_{1}^{\prime \prime}$ and $T_{3} \sqsubseteq S_{3}^{\prime \prime}$;

Proposition 2.7. Suppose $\varepsilon_{1}=\left\langle S_{1}^{\prime}, S_{21}^{\prime}\right\rangle \vdash S_{1} \lesssim S_{2}$ and $\varepsilon_{2}=\left\langle S_{22}^{\prime}, S_{3}^{\prime}\right\rangle \vdash S_{2} \lesssim S_{3}$. Then $\left(\varepsilon_{1}, \varepsilon_{2}\right)$ has a candidate for transitivity if and only if it has a least candidate over the precision (Б) order, denoted

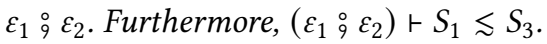


As Siek and Taha [2006] first observed, consistent subtyping is not transitive in general, and that property is fundamental to gradual type checking. However, transitivity of subtyping is fundamental

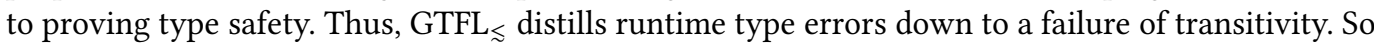
unlike the evidence inversion functions, consistent transitivity is a partial function.

Contextual reduction formalizes three kinds of reduction. First, it captures how notions of reduction apply in evaluation position. Second, it captures how an error during a notion of reduction aborts the entire computation. Third, it reflects how sequences of evidence objects are composed, producing new evidence on success or signaling a runtime type error on failure.

2.2.3 Type Safety. RL satisfies standard safety [Wright and Felleisen 1994]: the semantics explicitly categorizes all well-typed terms as reducible or as canonical forms. Programs do not get stuck, but they may signal runtime type errors. ${ }^{7}$

Proposition 2.8 (Progress [Garcia et al. 2016]). If $\mathbf{e} \triangleright \emptyset \vdash t: S$, then one of the following holds:

(1) e is a value $\mathbf{v}$;

(2) $\mathbf{e} \rightarrow \mathbf{e}^{\prime}$;

(3) $\mathrm{e} \rightarrow$ error.

Proposition 2.9 (Preservation [Garcia et al. 2016]). If $\mathbf{e} \triangleright \emptyset \vdash t: S$ and $\mathbf{e} \rightarrow \mathbf{e}^{\prime}$ then $\mathbf{e}^{\prime} \triangleright \emptyset \vdash t^{\prime}: S$ for some source $t^{\prime}$.

The statement of type preservation is unusual because the runtime typing judgment expresses a crisp relationship between source terms and runtime terms. The source terms $t$ in the runtime typing judgment evolve in lock-step with the runtime terms e. We say more about this in Sec. 2.3

Unlike typical preservation theorems for languages with subtyping [Pierce 2002], the type of the resulting term remains exactly the same as the source term. This is critical because consistent subtyping does not denote safe substitutibility. ${ }^{8}$ Safety requires that any use of consistent subtyping must be mediated by runtime evidence. Even if a subterm does evolve to a consistent subtype, it will be wrapped with runtime evidence that explicitly coerces it back to the original consistent supertype.

\subsection{Elaboration}

$\mathrm{GTFL}_{\lessgtr}$ source programs are elaborated to RL programs by type-directed translation (Fig. 4). The elaboration process is quite uniform, exhibiting the tight connection between $\mathrm{GTFL}_{\lessgtr}$ and RL. In essence RL terms represent $\mathrm{GTFL}_{\lessgtr}$ derivation trees, adding essential runtime type information (i.e. evidence) and erasing superfluous source type information.

Each elaboration rule corresponds directly to a source $\mathrm{GTFL}_{\lessgtr}$ typing rule. In particular, each premise typing judgment becomes a corresponding elaboration judgment, and each consistent subtyping judgment introduces an evidence object, using the initial evidence operator. ${ }^{9}$ The initial evidence operator $\mathcal{I}\left[\left[S_{1} \lesssim S_{2}\right]\right]$ computes the largest evidence object $\varepsilon \equiv\left\langle S_{1}^{\prime}, S_{2}^{\prime}\right\rangle$, such that $\left\langle S_{1}^{\prime}, S_{2}^{\prime}\right\rangle \vdash S_{1} \lesssim S_{2}$. From the definition of well-founded evidence in Sec. 2.2.1, we can deduce that $\left\langle S_{1}^{\prime}, S_{2}^{\prime}\right\rangle$ is the smallest pair of gradual types that subsumes all of the same static subtype instances $T_{1}<: T_{2}$ as $S_{1} \lesssim S_{2}$ does (i.e. if $T_{1}<: T_{2}$ then $\left\langle T_{1}, T_{2}\right\rangle \sqsubseteq\left\langle S_{1}, S_{2}\right\rangle$ iff $\left.\left\langle T_{1}, T_{2}\right\rangle \sqsubseteq\left\langle S_{1}^{\prime}, S_{2}^{\prime}\right\rangle\right)$. Naturally, initial evidence is undefined if $S_{1} \nless S_{2}$, but that circumstance does not arise if type checking succeeds.

The ( $\sim$ if) and ( $\sim$ app) elimination rules produce evidence for extra consistent subtyping judgments that were not evident in the corresponting (Sif) and (Sapp) GTFL $\mathrm{S}_{\S}$ rules. For instance,

\footnotetext{
${ }^{7} \mathrm{GTFL}_{\lessgtr}$ RL's safety follows from how its dynamic semantics were calculated, using AGT, from $\mathrm{STFL}_{<}$'s safety proof.

${ }^{8}$ This is most evident in the fact that consistent subtyping is not transitive.

${ }^{9} \mathrm{An}$ inductive definition of initial evidence is presented in the accompanying technical report [Bañados Schwerter et al 2020], see Fig. 14
} 
$\Gamma \vdash t \sim \mathbf{e}: S \quad$ Elaboration

$$
\begin{aligned}
& (\sim \mathrm{x}) \frac{x: S \in \Gamma}{\Gamma \vdash x \sim \mathrm{x}: S} \\
& (\sim \mathrm{n}) \frac{}{\Gamma \vdash n \sim \mathbf{n}: \operatorname{lnt}} \\
& (\sim \mathrm{b}) \frac{}{\Gamma \vdash b \sim \mathbf{b}: \text { Bool }} \\
& \Gamma \vdash t_{1} \leadsto \mathbf{e}_{1}: S_{1} \quad \varepsilon_{1}=\mathcal{I}\left[\left[S_{1} \lesssim \widetilde{\operatorname{dom}}\left(S_{1}\right) \rightarrow \widetilde{\operatorname{cod}}\left(S_{1}\right)\right]\right] \\
& (\sim \text { app }) \frac{\Gamma \vdash t_{2} \sim \mathbf{e}_{2}: S_{2} \quad \varepsilon_{2}=\mathcal{I}\left[\left[S_{2} \lesssim \widetilde{\operatorname{dom}}\left(S_{1}\right)\right]\right]}{\Gamma \vdash t_{1} t_{2} \leadsto \varepsilon_{1} \mathbf{e}_{1} \varepsilon_{2} \mathbf{e}_{2}: \widetilde{\operatorname{cod}}\left(S_{1}\right)} \\
& (\sim+) \frac{\overline{\Gamma \vdash t_{i} \sim \mathbf{e}_{i}: S_{i}} \quad \overline{\varepsilon_{i}=\mathcal{I}\left[\left[S_{i} \lesssim \operatorname{Int}\right]\right.}}{\Gamma \vdash t_{1}+t_{2} \sim \varepsilon_{1} \mathbf{e}_{1}+\varepsilon_{2} \mathbf{e}_{2}: \operatorname{lnt}} \\
& (\sim \operatorname{proj}) \frac{\Gamma \vdash t \sim \mathbf{e}: S \quad \varepsilon=\mathcal{I}[[S \lesssim[l: \widetilde{\operatorname{proj}}(S, l)]]]}{\Gamma \vdash t . l \sim \varepsilon \mathbf{e} . l: \widetilde{\operatorname{proj}}(S, l)} \\
& (\sim::) \frac{\Gamma \vdash t \leadsto \mathbf{e}: S \quad \varepsilon=\mathcal{I}\left[\left[S \lesssim S_{1}\right]\right]}{\Gamma \vdash\left(t:: S_{1}\right) \leadsto \varepsilon \mathbf{e}: S_{1}}
\end{aligned}
$$

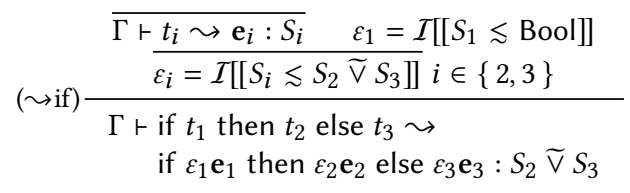

$$
\begin{aligned}
& (\sim \lambda) \frac{\Gamma, x: S_{1} \vdash t \leadsto \mathbf{e}: S_{2}}{\Gamma \vdash\left(\lambda x: S_{1} . t\right) \sim(\lambda \mathbf{x} . \mathbf{e}): S_{1} \rightarrow S_{2}} \\
& (\sim \mathrm{rec}) \frac{\overline{\Gamma \vdash t_{i} \sim \mathrm{e}_{i}: S_{i}}}{\Gamma \vdash\left[\overline{l_{i}=t_{i}}\right] \leadsto\left[\overline{l_{i}=\mathrm{e}_{i}}\right]:\left[\overline{l_{i}: S_{i}}\right]}
\end{aligned}
$$

Fig. 4. $\mathrm{GTFL}_{\lesssim}$ : Elaboration

$\left(\sim\right.$ app) demands that $S_{1} \lesssim \widetilde{\operatorname{dom}}\left(S_{1}\right) \rightarrow \widetilde{\operatorname{cod}}\left(S_{1}\right)$. This extra constraint was implied by the GTFL requirement that $\operatorname{cod}\left(S_{1}\right)$ be well-defined. The elaboration rule, and the corresponding RL term, make this implicit constraint explicit because it must be enforced at runtime. Similarly, the ( $\sim$ if) type $S_{2} \widetilde{V} S_{3}$ imposes implicit consistent subtyping constraints on each branch of the conditional.

The tight connection between $\mathrm{GTFL}_{\S}$ and $\mathrm{RL}$ is confirmed by preservation of well-typedness.

Proposition 2.10 (Well-formed Translation). If $\Gamma \vdash t \leadsto \mathbf{e}: S$ then $\mathbf{e} \vdash \Gamma \vdash t: S$.

So the source term $t$ elaborates to a runtime term e that represents $t$ 's static typing derivation.

We are now equipped to better explain the statement of preservation in Prop. 2.9. We relate runtime terms to source terms using the runtime typing judgment $\mathrm{e} \triangleright \Gamma \vdash t: S$ and source terms to runtime terms using the translation judgment $\Gamma \vdash t \leadsto \mathbf{e}: S$. Preservation clarifies how runtime terms "learn" new type constraints that are not evident in source programs.

$$
\text { Proposition } 2.11 \text { (Replicant). If } \mathbf{e} \triangleright \Gamma \vdash t: S \text { and } \Gamma \vdash t \leadsto \mathbf{e}^{\prime}: S \text { then } \mathbf{e} \sqsubseteq \mathbf{e}^{\prime} \text {. }
$$

In this proposition, $\mathbf{e} \sqsubseteq \mathbf{e}^{\prime}$ refers to the pointwise extension of evidence precision from types to terms. This proposition says that a runtime term e embodies at least as many runtime type constraints as any source term that it represents. In the above term, we call $\mathbf{e}^{\prime}$ a replicant of $\mathbf{e}$, because it amounts to cloning the structure of e but omitting its "memory" of any additional type constraints acquired while evaluating the program. The $\mathrm{e}^{\prime}$ term starts with a clean slate, yet to be jaded by the tribulations of runtime type enforcement.

The key idea is that a source program contains enough local type information to justify its plausible typeability, but the runtime term e must account for type invariants imposed at runtime. Below, we observe that these discovered type constraints must be represended precisely in order to enforce type-based invariants at runtime in mixed-type programs. 


\subsection{GTFL $_{\lesssim}$ Shortcomings Revisited}

Using the semantics of $\mathrm{GTFL}_{\lesssim}$ presented above, we can more closely examine the program from Sec. 1 that failed to protect type invariant boundaries. Our example program:

$$
\text { let } \mathrm{q}:[\mathrm{x}: \operatorname{lnt}]=[\mathrm{x}=5, \mathrm{y}=\operatorname{true}] \text { in }(\mathrm{q}:: \text { ?:: }[\mathrm{x}: \ln \mathrm{t}, \mathrm{y}: \text { Bool }]) \cdot \mathrm{y}
$$

elaborates to the following: ${ }^{10}$

$$
\text { let } \mathrm{q}=\varepsilon_{1}[\mathrm{x}=5, \mathrm{y}=\text { true }] \text { in } \varepsilon_{4}\left(\varepsilon_{3} \varepsilon_{2} q\right) \cdot \mathrm{y}
$$

where

$$
\begin{aligned}
\varepsilon_{1} & =\mathcal{I}[[[\mathrm{x}: \operatorname{Int}, \mathrm{y}: \text { Bool }] \lesssim[\mathrm{x}: \operatorname{Int}]]] & & =\langle[\mathrm{x}: \operatorname{Int}, \mathrm{y}: \text { Bool }],[\mathrm{x}: \operatorname{lnt}]\rangle \\
\varepsilon_{2} & =\mathcal{I}[[[\mathrm{x}: \operatorname{Int}] \lesssim ?]] & & =\langle[\mathrm{x}: \operatorname{Int}],[?]\rangle \\
\varepsilon_{3} & =\mathcal{I}[[? \lesssim[\mathrm{x}: \operatorname{Int}, \mathrm{y}: \text { Bool }]]] & & =\langle[\mathrm{x}: \operatorname{Int}, \mathrm{y}: \text { Bool, } ?],[\mathrm{x}: \operatorname{Int}, \mathrm{y}: \text { Bool }]\rangle \\
\varepsilon_{4} & =\mathcal{I}[[[\mathrm{x}: \operatorname{Int}, \mathrm{y}: \text { Bool }] \lesssim[\mathrm{y}: \text { Bool }]]] & & =\langle[\mathrm{x}: \operatorname{Int}, \mathrm{y}: \text { Bool }],[\mathrm{y}: \text { Bool }]\rangle
\end{aligned}
$$

Evaluation proceeds as follows:

$$
\begin{aligned}
& \text { let } \mathrm{q}=\varepsilon_{1}[\mathrm{x}=5, \mathrm{y}=\text { true }] \text { in } \varepsilon_{4}\left(\varepsilon_{3} \varepsilon_{2} q\right) \cdot \mathrm{y} \quad \longrightarrow \varepsilon_{4}\left(\varepsilon_{3} \varepsilon_{2} \varepsilon_{1}[\mathrm{x}=5, \mathrm{y}=\text { true }]\right) \cdot \mathrm{y}
\end{aligned}
$$

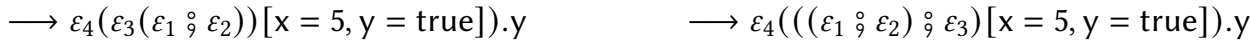

$$
\begin{aligned}
& \longrightarrow\left(\left(\left(\varepsilon_{1} \circ \varepsilon_{2}\right) \stackrel{\circ}{\circ} \varepsilon_{3}\right) \circ \varepsilon_{4}\right)[\mathrm{x}=5, \mathrm{y}=\text { true }] . \mathrm{y} \quad \longrightarrow \varepsilon_{5} \text { true }
\end{aligned}
$$

where

$$
\begin{aligned}
& \left(\varepsilon_{1} \circ \varepsilon_{2}\right)=\langle[\mathrm{x}: \operatorname{lnt}, \mathrm{y}: \mathrm{Bool}],[?]\rangle \quad\left(\left(\left(\varepsilon_{1} \stackrel{\circ}{9} \varepsilon_{2}\right) \stackrel{\circ}{q} \varepsilon_{3}\right) \stackrel{\circ}{q} \varepsilon_{4}\right)=\langle[\mathrm{x}: \text { Int, } \mathrm{y}: \mathrm{Bool}],[\mathrm{y}: \mathrm{Bool}]\rangle
\end{aligned}
$$

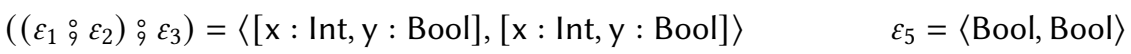

This program should signal a run-time type error, but it does not. Tracing its evaluation reveals what went wrong. First, observe that $\varepsilon_{2} \stackrel{\circ}{9} \varepsilon_{3}$ is undefined, even though $\left(\left(\varepsilon_{1} q_{9} \varepsilon_{2}\right) \circ \varepsilon_{3}\right)$ is defined. This demonstrates that composition is not associative. Now consider $\left(\varepsilon_{1} \circ \varepsilon_{2}\right)=\langle[\mathrm{x}: \operatorname{lnt}, \mathrm{y}: \mathrm{Bool}],[?]\rangle$ more closely. Appealing to our notion of well-formed evidence, this object contains via precision, every valid static subtyping pair $[\mathrm{x}:$ Int, $\mathrm{y}:$ Bool $]<: T$, including $T=[\mathrm{x}: \operatorname{lnt}, \mathrm{y}:$ Bool $]$.

However, if we consider the correctness criteria for Prop. 2.7, as applied to composing $\varepsilon_{1}$ with $\varepsilon_{2}$, then the only static types that complete the relevant triples $T_{1}<: T_{2}<: T_{3}$ are $T_{3}=[\mathrm{x}: \operatorname{Int}]$ and $T_{3}=[$ ]. Thus [ $\mathrm{x}:$ Int, $\mathrm{y}: \mathrm{Bool}]$ is a spurious potential supertype due solely to the choice of evidence abstraction. These phenomena suggest that our abstraction for evidence is insufficiently precise. We fix this problem in Sec. 5.

\section{THE ESSENCE OF AGT}

Since our goal is to develop a general solution to the demonstrated issues, our approach must be couched in terms of the AGT framework itself. This section presents a brief introduction to the AGT methodology and its basis in abstract interpretation [Cousot and Cousot 1977]. We reconceive some of the concepts involved in a manner that is equivalent to the original. Our new approach improves on the original in that it clarifies the nature of $t w o$ distinct but related abstractions: one for the static semantics and one for the dynamic semantics. This refinement is critical to our contributions. Along the way, we explain relevant aspects of AGT that we suppressed in Sec. 2.

Given a pre-existing statically typed language and its type safety argument, AGT starts by extending the syntax of types to form gradual types, in our case $S \in$ GType. In addition to the syntax for gradual types, we endow gradual types with a semantics: each gradual type $S$ is given

\footnotetext{
${ }^{10}$ The typing and elaboration rules for let can be deduced from the rules for lambda and application.
} 
an interpretation as a non-empty set of static types from the static language, denoted $\mathcal{P}^{+}$(Type). Following abstract interpretation terminology, this function is called concretization.

$$
\begin{gathered}
\gamma: \text { GType } \rightarrow \mathcal{P}^{+}(\text {Type }) \\
\gamma(S)=\{T \in \text { Type } \mid T \sqsubseteq S\} .
\end{gathered}
$$

Thus, a gradual type denotes every static type that it could represent. Since every static type is a (minimal) gradual type, this implies that $\gamma(T)=\{T\}$. When starting from scratch, concretization is typically defined by recursion on the structure of gradual types [Garcia et al. 2016], and then the precision relation is determined by the following correctness criterion:

Proposition 3.1 ([Garcia et al. 2016]). $S_{1} \sqsubseteq S_{2}$ if and only if $\gamma\left(S_{1}\right) \subseteq \gamma\left(S_{1}\right)$.

Here we reverse the development, defining concretization in terms of precision, since precision is introduced in Sec. 2. These approaches are equivalent, though a structurally recursive definition of concretization is critical to calculating functions and relations on gradual types like $\widetilde{\text { proj }}$ and $\mathcal{I}$.

The concretization function in turn induces a converse function, typically called abstraction.

$$
\begin{gathered}
\left.\alpha: \mathcal{P}^{+} \text {(Type }\right) \rightarrow \text { GType } \\
\alpha(C)=\prod\{S \in \text { GType } \mid C \subseteq \gamma(S)\}
\end{gathered}
$$

In this definition, $\prod$ denotes the greatest lower-bound with respect to precision $\sqsubseteq$. Abstraction $\alpha(C)$ yields the most precise gradual type that summarizes a given set of static types. In order for $\alpha$ to be well-defined, we must ensure that the right-hand side of the above equation is well-defined for any collection $C$ to be considered: in particular there must exist an appropriate greatest lower-bound. In general, a gradual type system does not need to account for every arbitrary subset of static types $C \in \mathcal{P}^{+}$(Type) as part of concretization and abstraction. In fact, in Sec 5.3 it will be critical to restrict the space of sets of types (with no loss of expressiveness) to ensure that $\alpha$ is well-defined. ${ }^{11}$ If abstraction $\alpha$ is well-defined, then it is uniquely determined by concretization $\gamma$. To facilitate the calculation of operators and relations on gradual types, abstraction is typically presented as a recursive function definition [Garcia et al. 2016].

By design, concretization and abstraction are related by two properties that together characterize a Galois connection [Cousot and Cousot 1977].

Proposition 3.2 ([Garcia et al. 2016]).

(1) Soundness: $C \subseteq \gamma(\alpha(C))$

(2) Optimality: If $C \subseteq \gamma(S)$ then $\gamma(\alpha(C)) \subseteq \gamma(S)$.

These functions satisfy the stronger property that $\alpha(\gamma(S))=S$. This makes them a Galois insertion.

Garcia et al. [2016] use concretization and abstraction to define all of the gradual typing artifacts used to define $\mathrm{GTFL}_{\varsigma}$. For example, consider the codomain of a function type, rendered as a partial function $\operatorname{cod}$ on static types:

$$
\begin{gathered}
\operatorname{cod}: \text { Type } \rightarrow \text { Type } \\
\operatorname{cod}\left(T_{1} \rightarrow T_{2}\right)=T_{2} \\
\operatorname{cod}(T) \text { undefined otherwise }
\end{gathered}
$$

\footnotetext{
${ }^{11}$ Unlike Garcia et al. [2016], we define $\alpha$ to be a total function over a family of (non-empty) sets of types, rather than a partial function over arbitrary sets of types $\mathcal{P}$ (Type). This approach pushes partiality into the collecting operators $\left(c f\right.$. cod $d^{+}$ below), rather than the Galois connection itself. We find this approach more general, intuitive, and mathematically pleasant.
} 
We can idiomatically lift cod to a partial function $\operatorname{cod}^{+}$on non-empty sets of types.

$$
\begin{gathered}
\operatorname{cod}^{+}: \mathcal{P}^{+}(\text {Type }) \rightarrow \mathcal{P}^{+}(\text {Type }) \\
\operatorname{cod}^{+}(C)=C^{\prime} \text { if } C^{\prime} \neq \emptyset \\
\operatorname{cod}^{+}(C) \text { undefined if } C^{\prime}=\emptyset \\
\text { where } \quad C^{\prime}=\left\{T^{\prime} \in \text { Type } \mid T \in C \text { and } \operatorname{cod}(T)=T^{\prime}\right\}
\end{gathered}
$$

The $\operatorname{cod}^{+}$function applies cod pointwise to a set of types, and produces the resulting set of codomains, so long as there are some codomains: if not, then the function is undefined. Using this lifted function, we can define the $\widetilde{c o d}$ function as follows.

$$
\widetilde{\operatorname{cod}}(S)=\alpha\left(\operatorname{cod}^{+}(\gamma(S)) .\right.
$$

Given the definitions of $\widetilde{\operatorname{cod}}, \gamma$, and $\alpha$, the correctness criteria of Prop. 2.2 follow. From there, the recursive characterization of $\widetilde{c o d}$ from Fig. 1 can be calculated by induction on the structure of $S$. Analogous constructions can be used to first define and then calculate algorithms for all of the operations on gradual types from Sec. 2. The definitions directly imply their own correctness criteria, and the corresponding algorithms require tedious but straightforward calculations.

Evidence Is Its Own Abstraction. The concept of evidence for consistent subtyping arises through an analogous process. Garcia et al. [2016] define evidence by abstracting tuples of static types, filtering them post-hoc. Our approach refines theirs in a small but fundamental way. In particular, we introduce a second Galois connection, but this one is directly between consistent subtyping, regarded as a set of pairs of gradual types $\left\langle S_{1}, S_{2}\right\rangle \in \lesssim$, and nonempty subsets of the static subtyping relation, i.e. sets of pairs $\mathcal{R} \subseteq<$ :

The key difference in this new approach is that the set of evidence objects form in their entirety an abstract interpretation of static subtyping. In contrast, the evidence objects introduced by [Garcia et al. 2016] were arbitrary pairs of gradual types. The concretization of an evidence object could therefore contain pairs of types not related by subtyping. Integrating subtyping into the conception of abstraction, and viewing composition as relational composition specifically for fragments of subtyping is critical to formulating the possibility, let alone the significance, of forward completeness.

We begin with concretization.

$$
\begin{gathered}
\gamma^{<:}: \lesssim \rightarrow \mathcal{P}^{+}(<:) \\
\gamma^{<:}\left(S_{1}, S_{2}\right)=\left\{\left\langle T_{1}, T_{2}\right\rangle \mid T_{i} \in \gamma\left(S_{i}\right) \text { and } T_{1}<: T_{2}\right\} .
\end{gathered}
$$

The corresponding notion of abstraction is defined as follows

$$
\begin{gathered}
\alpha^{<:}: \mathcal{P}^{+}(<:) \rightarrow \lesssim \\
\alpha^{<:}(\mathcal{R})=\left\langle\alpha\left(\pi_{1}(\mathcal{R})\right), \alpha\left(\pi_{2}(\mathcal{R})\right)\right\rangle .
\end{gathered}
$$

Abstraction appeals to two point-wise projection functions that operate on sets of pairs. These recur in later developments.

$$
\begin{gathered}
\pi_{1}, \pi_{2}: \mathcal{P}\left(\text { Type }^{2}\right) \rightarrow \mathcal{P}(\text { Type }) \\
\pi_{i}\left(\left\{\overline{\left\langle T_{1}, T_{2}\right\rangle}\right\}\right)=\left\{\overline{T_{i}}\right\}
\end{gathered}
$$

The functions $\gamma^{<:}$and $\alpha^{<:}$form a Galois connection, but they do not form a Galois insertion. For example: $\alpha^{<:}\left(\gamma^{<:}(\operatorname{Int}, ?)\right)=\langle\operatorname{Int}$, Int $\rangle$. In fact, $\alpha^{<:}\left(\gamma^{<:}\left(S_{1}, S_{2}\right)\right)$ is equivalent, by design, to the initial evidence function $\mathcal{I}\left[\left[S_{1} \lesssim S_{2}\right]\right]$ from Fig. 4 . The $\mathcal{I}$ function simply extends its domain to accept, but 
be undefined for, inconsistent gradual types $S_{1} \npreceq S_{2}$. Thus, evidence objects $\varepsilon$ are exactly those pairs of gradual types in the image of $\alpha^{<:} \circ \gamma^{<:}$.

Furthermore, composing evidence objects using the consistent transitivity operator can be defined in terms of relational composition of subsets of static subtyping $<$ :

$$
\begin{gathered}
\varepsilon_{1} \stackrel{\circ}{9} \varepsilon_{2}=\alpha^{<:}\left(I d^{+}\left(\gamma^{<:}\left(\varepsilon_{1}\right) ; \gamma^{<:}\left(\varepsilon_{2}\right)\right)\right) \\
\text { where } \mathcal{R}_{1} ; \mathcal{R}_{2}=\left\{\left\langle T_{1}, T_{3}\right\rangle \mid T_{1} \mathcal{R}_{1} T_{2} \text { and } T_{2} \mathcal{R}_{2} T_{3} \text { for some } T_{2}\right\} \\
\text { and } I d^{+}(\mathcal{R})= \begin{cases}\mathcal{R} & \mathcal{R} \neq \emptyset \\
\text { undefined } & \text { otherwise. }\end{cases}
\end{gathered}
$$

This definition can be used to calculate a direct recursive characterization of consistent transitivity (see technical report [Bañados Schwerter et al. 2020]), but we can also prove that it is equivalent to a combination of simpler operations. ${ }^{12}$

Proposition 3.3.

$$
\left\langle S_{1}, S_{21}\right\rangle_{9}\left\langle S_{22}, S_{3}\right\rangle=\mathcal{I}\left[\left[\pi_{1}\left(\mathcal{I}\left[\left[S_{1} \lesssim\left(S_{21} \sqcap S_{22}\right)\right]\right]\right) \lesssim \pi_{2}\left(\mathcal{I}\left[\left[\left(S_{21} \sqcap S_{22}\right) \lesssim S_{3}\right]\right]\right)\right]\right]
$$

where $S_{21} \sqcap S_{22}=\alpha\left(I d^{+}\left(\gamma\left(S_{21}\right) \cap \gamma\left(S_{22}\right)\right)\right)$.

A key observation here is that pairs of gradual types are but one arbitrary, albeit convenient, abstraction for subsets of the static subtyping relation. This analysis of evidence as abstractions of static subtyping fragments, and consistent transitivity as abstract relational composition, is critical to our analysis and improvement of evidence in Sec. 5.

\section{SPACE EFFICIENT SEMANTICS FOR AGT-BASED LANGUAGES}

The dynamic semantics presented in Fig. 3 follows the standard AGT methodology. Evidence is composed after an ascribed expression is reduced, in an order that accumulates evidence on a resulting value. Thus RL, and every other AGT-based language, suffers from the same space issues described by Herman et al. [2010]. This section presents an alternate reduction semantics, $\mathrm{RL}^{+}$, that composes evidence as soon as possible to prevent the accumulation of pending evidence objects $\mathrm{RL}^{+}$requires associative evidence composition to guarantee that it will evaluate to the same results as RL. Unfortunately, this property does not hold for $\mathrm{GTFL}_{\varsigma}$, but Sec. 5 addresses this limitation by providing a better evidence abstraction. Sec. 6 describes criteria that guarantee associativity for evidence composition. Sec. 7 proves that the criteria hold for our novel abstraction, and proves an upper-bound on space consumption.

Space-Efficient, But Observationally Equivalent. To preserve tail recursion, a semantics must compose evidence eagerly, thereby keeping evidence objects from accumulating in tail position. However, to keep the semantics equivalent to the original, the space-efficient one must not fail early in case a program diverges. To preserve this behavior, a space-efficient semantics must remember "latent failure" until evaluation yields a value, while avoiding unbounded stack growth.

The $\mathrm{RL}^{+}$semantics applies the standard technique of explicitly representing failure. It introduces "total" evidence, $\varepsilon_{\perp}$, which represents either normal evidence $\varepsilon$, or a latent failure $\perp$, which serves the same purpose as the failure coercion of [Herman et al. 2010]. A latent failure indicates that some evidence composition was undefined, and will trigger an error after its argument finishes reducing. The syntax for $\mathrm{RL}^{+}$terms (Fig. 5), essentially mirrors RL, but substitutes total evidence for evidence. To emphasize this distinction, we color the terms in the $\mathrm{RL}^{+}$differently e.g., $e$.

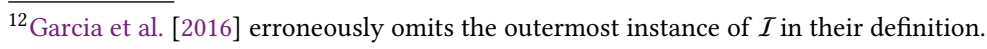


Syntax

$$
\begin{aligned}
& \varepsilon_{\perp}::=\perp \mid \varepsilon \quad \text { (total evidence) } \\
& e \in \mathrm{RTerm} \quad::=n\left|\varepsilon_{\perp} e+\varepsilon_{\perp} e\right| x|\lambda x . e| \varepsilon_{\perp} e \varepsilon_{\perp} e\left|\varepsilon_{\perp} e\right|[\overline{l=e}] \mid \varepsilon_{\perp} e . l \quad \text { (runtime terms) } \\
& \text { | if } \varepsilon_{\perp} e \text { then } \varepsilon e \text { else } \varepsilon e \\
& F \in \text { EvFrame ::= } \square \varepsilon e|\varepsilon u \square| \square+\varepsilon e|\varepsilon u+\square| \square . l \quad \text { (runtime frames) } \\
& \text { । if } \square \text { then } \varepsilon e \text { else } \varepsilon e \\
& G \in \operatorname{ACtxt}::=\quad \square \mid E[F[\square] \mid E[[\overline{l=\mathrm{v}}, l=\square, \overline{l=\mathrm{e}}]] \quad \text { (Evaluation contexts } \\
& \text { without an innermost ascription) } \\
& \text { (Evaluation contexts) }
\end{aligned}
$$

Contextual Reduction

$e \longrightarrow e, e \longrightarrow$ error

$$
\frac{e \leadsto e^{\prime}}{E[e] \longrightarrow E\left[e^{\prime}\right]} \quad \frac{e \leadsto \text { error }}{E[e] \longrightarrow \text { error }} \quad \frac{}{G\left[\varepsilon_{\perp 1} \varepsilon_{\perp 2} e\right] \longrightarrow G\left[\varepsilon_{\perp 2} \stackrel{\circ}{\perp}_{\perp} \varepsilon_{\perp 1} e\right]} \quad \frac{}{G[\perp u] \longrightarrow \text { error }}
$$

Fig. 5. Syntax and Contextual Reduction of $\mathrm{RL}^{+}$. All other parts of the semantics are as in RL.

One key difference in the definition of $\mathrm{RL}^{+}$is its runtime frames: the empty $\square$ frame, which allows evidence objects to accumulate on the RL evaluation stack, is omitted. Frames otherwise remain the same: For example, $\mathrm{RL}^{+}$does not need a frame of the form $\varepsilon_{\perp} u \square$ because when the function position of an application reduces to $\perp u$, the original semantics would trigger an error.

\subsection{The Reduction Semantics of $\mathbf{R L}^{+}$}

$\mathrm{RL}^{+}$has the same the notions of reduction as RL. Thus, it suffices to change the definition of contextual reduction $\longrightarrow$. To compose evidence objects ahead of time without failing too early,

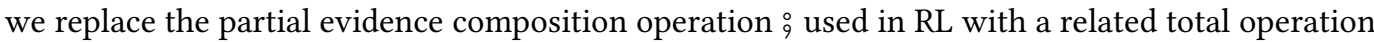
denoted $\stackrel{\circ}{\perp}_{\perp}$, which yields latent failures $\perp$ when $\stackrel{9}{\text { is undefined: }}$

$$
\varepsilon_{\perp 1} \stackrel{\circ}{\perp} \perp_{\perp 2} \varepsilon_{\perp 2}= \begin{cases}\varepsilon_{1} \stackrel{\circ}{9} \varepsilon_{2} & \text { if } \varepsilon_{\perp 1}=\varepsilon_{1} \text { and } \varepsilon_{\perp 2}=\varepsilon_{2} \text { and composition is defined } \\ \perp & \text { otherwise }\end{cases}
$$

Since the definition of frames no longer includes holes, ascriptions can no longer nest in evaluation contexts. In fact, evidence objects that come from ascriptions, rather than elimination forms, do not form evaluation contexts: they must be handled explicitly. Composition no longer waits for values, but proceeds when nested ascriptions become the immediate redex.

Finally, to preserve divergent behaviour, errors are triggered by notions of reduction only upon reaching a value $(G[\perp u])$.

We prove that $\mathrm{RL}$ and $\mathrm{RL}^{+}$are weakly bisimilar. Fig. 6 introduces a bisimulation relation between terms in the both languages to carry the proof. While most rules follow inductively, rule [Sim-Casts] accepts terms with differing stacks of evidence compositions, as long as the final composed result (in each language's order) is the same.

Theorem 4.1 (Weak Bisimulation between $\mathrm{RL}$ and $\mathrm{RL}^{+}$). If $e_{1} \approx e_{2}{ }^{13}$ and evidence composition is associative, then:

- If there exists $e_{1}^{\prime}$ in $R L$ such that $e_{1} \longrightarrow e_{1}^{\prime}$, then there exists $e_{2}^{\prime}$ such that $e_{2} \rightarrow^{*} e_{2}^{\prime}$ and $e_{1}^{\prime} \approx e_{2}^{\prime}$.

- If there exists $e_{2}^{\prime}$ in $R L^{+}$such that $e_{2} \longrightarrow e_{2}^{\prime}$, then there exists $e_{1}^{\prime}$ such that $e_{1} \rightarrow^{*} e_{1}^{\prime}$ and $e_{1}^{\prime} \approx e_{2}^{\prime}$. Therefore, the relation $\approx$. forms a Weak Bisimulation [Sangiorgi 2011] between $\longrightarrow$ and $\longrightarrow$.

\footnotetext{
${ }^{13}$ The proof of the theorem appears in Sec. D.3 of the technical report [Bañados Schwerter et al. 2020].
} 
$e \approx e$, error $\approx$ error

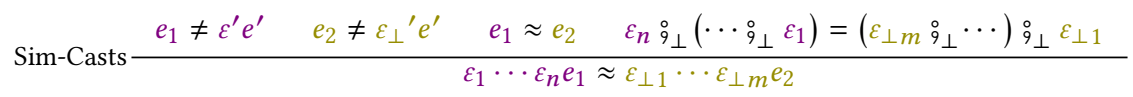

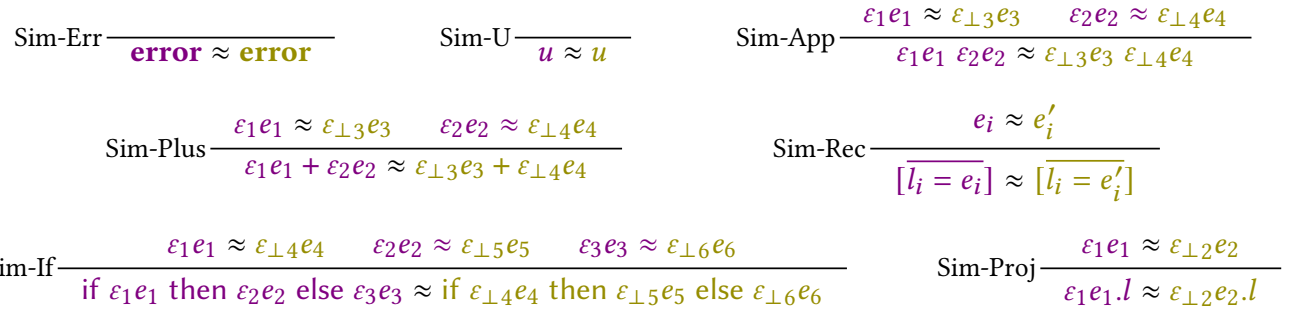

Fig. 6. Bisimulation Relation for space efficiency

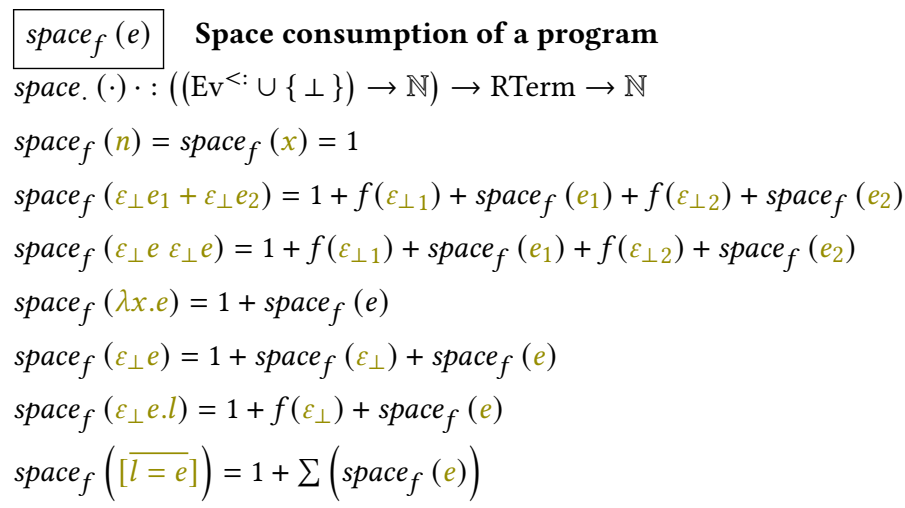

Fig. 7. Space consumption of a program in $\mathrm{RL}^{+}$

\subsection{Claiming Space-Efficiency for $\mathrm{RL}^{+}$}

The $\mathrm{RL}^{+}$dynamic semantics never accumulate evidence compositions in tail position, but this alone does not guarantee space efficiency. If evidence objects themselves can grow without bound, then the changes to the semantics amount to simply shuffling space usage without eradicating it. To ensure space efficiency we must quantify the overhead of evidence throughout evaluation.

Following Herman et al. [2010], we provide a bound for the space consumption of a program. The ppace $_{f}(e)$ function (Fig. 7) is parameterized by a function $f$ for the space consumption of an evidence object. We use this structural function to compare the space consumption of a program with "0-cost” evidence to $\mathrm{RL}^{+}$programs.

Definition 4.2 (Space-efficiency of evidence). Let size be the function that computes the space consumption of an evidence object. A reduction semantics is space efficient with respect to evidence objects if the overhead due to carrying evidence is bound throughout evaluation and ascriptions never wait on the stack:

- Bound for overhead. There exists a bound function $B:$ RTerm $\rightarrow \mathbb{N}$ such that, for any programs $e$ and $e^{\prime}$ such that $e \longrightarrow{ }^{*} e^{\prime}$, space $_{\text {size }}\left(e^{\prime}\right) \leq \operatorname{space}_{\lambda_{-} \cdot B(e)}\left(e^{\prime}\right)$.

This bound is equivalent to requiring that for any evidence $\varepsilon$ appearing in $e^{\prime}$, size $(\varepsilon) \leq B(e)$. 
- Bound for stack. For any programs $e$ and $e^{\prime}$ such that $e \longrightarrow e^{\prime}$, if there exists an evaluation context without nested ascriptions $G$ such that $e=G\left[\varepsilon \varepsilon^{\prime} e^{\prime \prime}\right]$, then there must exist an $\varepsilon^{\prime \prime}$ such that $e^{\prime}=G\left[\varepsilon^{\prime \prime} e^{\prime \prime}\right]$.

From this definition, a bound in the style of Herman et al. [2010] follows as a corollary:

Corollary 4.3 (Fixed overhead a-la-Herman et al. [2010]). If $e \longrightarrow^{*} e^{\prime}$ is bound for overhead with an evidence bound function $B$, then

$$
\text { space }_{\text {size }}\left(e^{\prime}\right) \leq 3 * B(e) * \text { space }_{0}\left(e^{\prime}\right)
$$

Proof. By induction over the structure of $e^{\prime}$.

Evidence requirements that make $\mathrm{RL}^{+}$space efficient. The development of space-efficient semantics can be completed with a new evidence abstraction for the $\mathrm{RL}^{+}$runtime semantics. Before introducing the abstraction, we present all the properties we need from this evidence. $\mathrm{RL}^{+}$semantics is space efficient for any abstraction whose evidence composition satisfies the following two properties:

Proposition 4.4 (Evidence composition is associative). For any evidence $\varepsilon_{\perp 1}, \varepsilon_{\perp 2}$ and $\varepsilon_{\perp 3}$,

$$
\left(\varepsilon_{\perp 1} \stackrel{\circ}{\perp}_{\perp} \varepsilon_{\perp 2}\right) \stackrel{\circ}{\circ}_{\perp} \varepsilon_{\perp 3}=\varepsilon_{\perp 1} \stackrel{\circ}{\perp}_{\perp}\left(\varepsilon_{\perp 2} \stackrel{\circ}{\perp}_{\perp} \varepsilon_{\perp 3}\right)
$$

Proposition 4.5 (Evidence composition has a bound). Let $\varepsilon_{1}$ and $\varepsilon_{2}$ be any evidence objects appearing in program e. For any evidence objects $\varepsilon_{1}^{\prime}$ and $\varepsilon_{2}^{\prime}$ such that $\operatorname{size}\left(\varepsilon_{1}^{\prime}\right) \leq \operatorname{size}\left(\varepsilon_{1}\right)$ and $\operatorname{size}\left(\varepsilon_{2}^{\prime}\right) \leq \operatorname{size}\left(\varepsilon_{2}\right), \operatorname{size}\left(\varepsilon_{1}^{\prime} \stackrel{9}{\varepsilon_{2}^{\prime}}\right) \leq B(e)$.

Theorem 4.6 (Properties of $\mathrm{RL}^{+}$). If evidence composition is associative and has a bound, then the semantics of $R L^{+}$is space-efficient and is observationally equivalent to the semantics of $R L$.

\section{PRECISE EVIDENCE FOR GTFL S $_{\lessgtr}$ BOUNDED RECORDS AND ROWS}

Previous sections identify the need for a more precise abstraction for runtime evidence. This section delivers that abstraction, and finds a natural characterization of precision that meets the paper's goals. We devise a notion of evidence that admits an associative consistent transitivity operation, and preserves the type invariants implied by the concrete fragments of static subtyping that each evidence object represents. This notion of evidence has some features in common with record-typing systems from the literature, though used here for runtime type checking, e.g. [Rémy 1989]. We replace gradual rows in evidence with a more precise gradual type abstraction, which we call bounded records and rows (BRR). We prove that evidence objects based on BRR are associative and precise using a general concept from abstract interpretation called forward completeness.

\subsection{Representing Optional Fields}

To motivate and introduce the structure of BRR, consider once again the failing example from Sec. 2.4. The key observation in that example is that in the following evidence composition,

$$
(\langle[\mathrm{x}: \operatorname{Int}, \mathrm{y}: \mathrm{Bool}],[\mathrm{x}: \operatorname{Int}]\rangle:\langle[\mathrm{x}: \operatorname{Int}],[?]\rangle)=\langle[\mathrm{x}: \operatorname{Int}, \mathrm{y}: \text { Bool }],[?]\rangle
$$

the second resulting gradual type represents too many possible static types, in particular the spurious cases $[y:$ Bool $]$ and $[x: \operatorname{lnt}, y:$ Bool $]$. A precise analysis of the two composed evidence objects admits only two possible supertypes: [ ] or [ $x: \operatorname{lnt}]$.

To faithfully capture this circumstance, BRR first introduces a distinction between required fields and optional fields. For instance, the BRR type $\left[\mathrm{x}: \operatorname{lnt}_{R}\right]$ uses the $R$ annotation to indicate that the $\mathrm{x}$ field is required: this BRR is equivalent to the $[\mathrm{x}: \operatorname{lnt}]$ gradual record type (and static record type). In contrast, the BRR type $\left[\mathrm{x}: \operatorname{Int} \mathrm{B}_{O}\right]$ uses the $O$ annotation to indicate that the $\mathrm{x}$ field is 
optional. Given the appropriate definition of concretization for BRR, we have []$\in \gamma([\mathrm{x}: \operatorname{lnt} O])$ and $[\mathrm{x}:$ Int $] \in \gamma\left(\left[\mathrm{x}: \operatorname{lnt} t_{O}\right]\right)$, and no other static type. This BRR type precisely captures the intended record types in the example above, and as we might expect, the above evidence and composition can be replaced by the precise BRR-based version:

$$
\left(\left\langle\left[\mathrm{x}: \operatorname{Int}_{R}, \mathrm{y}: \mathrm{Bool}_{R}\right],\left[\mathrm{x}: \operatorname{Int}_{R}\right]\right\rangle ;\left\langle\left[\mathrm{x}: \operatorname{Int}_{R}\right],\left[\mathrm{x}: \operatorname{Int}{ }_{O}\right]\right\rangle\right)=\left\langle\left[\mathrm{x}: \operatorname{Int}{ }_{R}, \mathrm{y}: \operatorname{Bool}_{R}\right],\left[\mathrm{x}: \operatorname{Int}{ }_{O}\right]\right\rangle
$$

Because the BRR abstraction is strictly more precise than the gradual row abstraction, the criteria set forth in Prop. 2.4 leads to more well-formed evidence objects. As a result, $\left\langle\left[\mathrm{x}: \operatorname{lnt} t_{R}\right],\left[\mathrm{x}: \operatorname{lnt} t_{O}\right]\right\rangle$ is well formed and, unlike gradual rows, it precisely captures the static subtype instances of interest.

\subsection{Representing Absent Fields}

Unfortunately, adding optional fields is not enough to achieve associativity. Consider the following trio of BRR-based evidence:

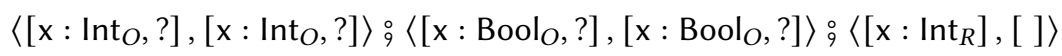

BRR must somehow represent rows to subsume the expressiveness of gradual row types. However, evaluating this composition reveals the need for more precise information. Composing left-to-right yields $\left\langle\left[\mathrm{x}: \operatorname{Int} t_{O}\right.\right.$, ?], $\left[\mathrm{x}: \operatorname{Int}_{O}\right.$, ?] $\rangle$ because composing the first two yields $\left\langle\left[\mathrm{x}: \operatorname{Int} t_{O}\right.\right.$, ?], [?] $\rangle$ which composes with the third to yield the above. On the other hand, composing them right-to-left fails! The reason is that the requirements of the second and third evidence objects are incompatible: the second can only have a Bool-typed $x$ field, while the third demands an Int-typed one.

Closer inspection of the first two evidence objects reveals that after composing them, the resulting evidence object should have no $\mathrm{x}$ field in the super type position: the only instances of static subtyping that could have transitively composed were those lacking an $\mathrm{x}$ field. Then the necessary absence of $x$ is incompatible with requiring $x$ to have Int type as in the third evidence.

This observation leads us to represent necessarily absent fields. Then the result of composing the first two evidence objects above is: $\left\langle\left[\mathrm{x}: \operatorname{Int}_{O}\right.\right.$, ?], $[\mathrm{x}: \varnothing$, ? $\left.]\right\rangle$. The BRR $[\mathrm{x}: \varnothing$, ?] represents the set of record types with any fields except $\mathrm{x}$. Composing this with $\left\langle\left[\mathrm{x}: \ln \mathrm{t}_{R}\right],[]\right\rangle$ should always fail. Adding absent and optional fields suffices to ensure that evidence objects need never lose information about plausible subtype relations, admitting implausible fields via the unknown row ?.

\subsection{Bounded Rows and Bounded Records, Formally}

We now give a full formal definition of Bounded Records and Rows (BRR) ${ }^{14}$, as well BRR-based evidence objects. The syntax of BRR is follows:

$$
M \in \text { Mapping }
$$

$$
\begin{aligned}
& M::=\varnothing\left|S_{R}\right| S_{O} \\
& S::=\text { Bool }|\operatorname{Int}| S \rightarrow S|?|\left[\sum_{i=1}^{n} \ell_{i}: M_{i}\right] \mid\left[\sum_{i=1}^{n} \ell_{i}: M_{i}, ?\right] \quad \text { (gradual types) }
\end{aligned}
$$

As its name implies, the only interesting aspects of the BRR abstraction are its record and row type definitions. Each field maps to a type (qualified as required $R$ or optional $O$ ), or to the absent field indicator $(\varnothing)$. Individual static record types are represented in BRR by simply marking all fields as required. BRR is a runtime abstraction, so its annotations do not affect $\mathrm{GTFL}_{\lesssim}$ syntax.

Though technically we need missing field annotations only for rows and not for records, we opt for a uniform representation. A mapping $\mathrm{x}: \varnothing$ in a record is redundant; similarly, a mapping $\mathrm{x}: ?_{O}$ is redundant in a row. This redundancy has algorithmic advantages: we can extract a default

\footnotetext{
${ }^{14}$ While not Rows in the sense of[Wand Mitchell '91], Bounded Rows are derived from Gradual Rows introduced by [Garcia et al. 2016]. We keep the Row designation to highlight the connection with the latter paper.
} 


\section{Decompositions}

$$
\begin{aligned}
& \left.C \in \mathcal{P} \text { (Type), } C^{\varnothing} \in \mathcal{P} \text { (Type } \cup\{\varnothing\}\right), d \in \text { Decomp } \\
& d::=\llbracket \text { Bool } \rrbracket|\llbracket \operatorname{lnt} \rrbracket| \llbracket C \rightarrow C \rrbracket|\llbracket ? \rrbracket| \llbracket \sum_{i=1}^{n} \ell_{i}: C^{\varnothing}{ }_{i} \rrbracket \mid \llbracket ? \sum_{i=1}^{n} \ell_{i}: C^{\varnothing} i \rrbracket \\
& \mathscr{C}:(\text { Decomp }) \rightarrow \mathcal{P}(\text { Type })
\end{aligned}
$$

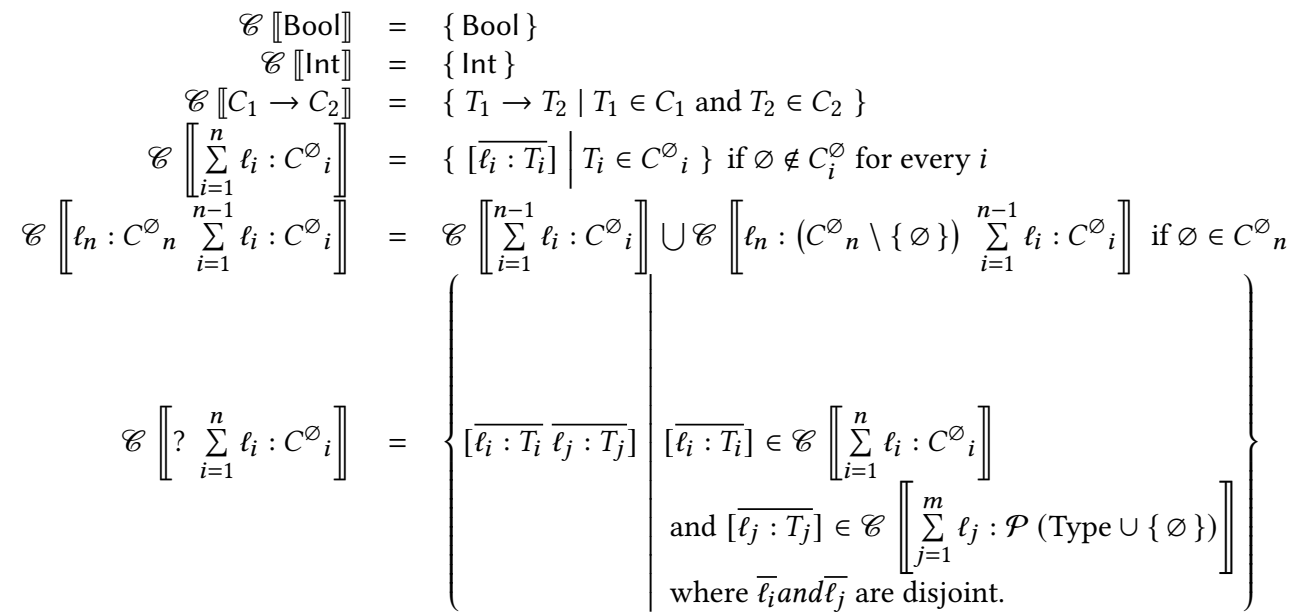

$$
\begin{aligned}
& \begin{aligned}
\gamma:(\text { GType }) \rightarrow \mathcal{P}^{+} \text {(Type) } \\
\gamma(?)=\text { Type }
\end{aligned} \\
& \gamma(\mathrm{Bool})=\mathscr{C} \llbracket \mathrm{Bool} \rrbracket \\
& \gamma(\operatorname{lnt})=\mathscr{C} \llbracket \ln t \rrbracket \\
& \gamma\left(S_{1} \rightarrow S_{2}\right)=\mathscr{C} \llbracket \gamma\left(S_{1}\right) \rightarrow \gamma\left(S_{2}\right) \rrbracket \\
& \gamma\left(\left[\sum_{i=1}^{n} \ell_{i}: M_{i} *\right]\right)=\mathscr{C} \llbracket * \sum_{i=1}^{n} \ell_{i}: \gamma^{M}\left(M_{i}\right) \rrbracket \\
& \begin{array}{l}
\gamma^{M}:(\text { Mapping }) \rightarrow \mathcal{P}^{+}(\text {Type } \cup\{\varnothing\}) \\
\gamma^{M}(\varnothing)=\{\varnothing\}
\end{array} \\
& \gamma^{M}\left(S_{R}\right)=\gamma(S) \\
& \gamma^{M}\left(S_{O}\right)=\gamma^{M}(\varnothing) \cup \gamma\left(S_{R}\right)
\end{aligned}
$$

Fig. 8. Decompositions and BRR Concretization Function

mapping for labels in either a row or a record, which simplifies inductive rules in those cases where the domains of two types differ.

The notation $\sum_{i=1}^{n} \ell_{i}: M_{i}$ is equivalent to the previous notation $\overline{\ell_{i}: M_{i}}$, but avoids confusion when trying to distinguish shared subdomains in two record-like types. Empty record and row types are still allowed (i.e. $n$ can be 0 ).

A concretization function (Fig. 8) determines the meaning of BRR types. An intermediate notion of decompositions simplifies our definitions. Decompositions encode uniformity restrictions on sets of static types. These restrictions are made explicit by use of the generator function $\mathscr{C}$.

The interesting equations are those for records, which recursively describe sets of records that meet the constraints described informally above. Note that the concretization of non-empty bounded records is described in terms of the concretization of smaller bounded records.

Fig. 9 defines the corresponding abstraction for bounded records and rows. The equations distinguish between relevant sets of static types. This abstraction function has a subtlety regarding its domain (which we do not explicitly name). The domain cannot be arbitrary sets of types. To 


$$
\begin{aligned}
& \alpha(\mathscr{C} \llbracket \text { Bool } \rrbracket)=\text { Bool } \\
& \alpha(\mathscr{C} \llbracket \operatorname{lnt} \rrbracket)=\operatorname{lnt} \\
& \alpha\left(\mathscr{C} \llbracket C_{1} \rightarrow C_{2} \rrbracket\right)=\alpha\left(C_{1}\right) \rightarrow \alpha\left(C_{2}\right) \\
& \alpha\left(\mathscr{C} \llbracket * \sum_{i=1}^{n} \ell_{i}: C^{\varnothing} \rrbracket\right)=\left[\sum_{i=1}^{n} \ell_{i}: \alpha^{M}\left(C^{\varnothing}{ }_{i}\right) *\right] \quad \begin{aligned}
\alpha^{M}(\{\varnothing\} \cup C) & =(\alpha(C))_{O} \text { if } C \text { is not empty } \\
\alpha^{M}(C) & =(\alpha(C))_{R} \text { if } \varnothing \notin C
\end{aligned} \\
& \alpha(\varnothing) \text { undefined } \\
& \alpha(C)=\text { ? otherwise }
\end{aligned}
$$

Fig. 9. BRR Abstraction Function

\section{$\vdash \varepsilon \mathrm{wf} \quad$ Well-formed Evidence}

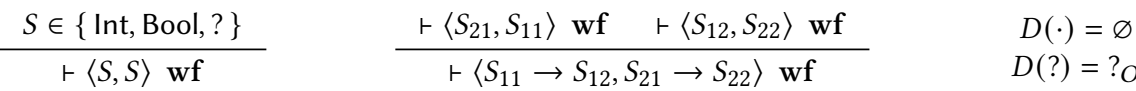

$$
\begin{aligned}
& \left\langle *_{1}, *_{2}\right\rangle \neq\langle\cdot, ?\rangle \quad \forall i, \vdash\left\langle M_{i 1}, M_{i 2}\right\rangle \text { wf } \quad \forall j, \vdash\left\langle M_{j}, D\left(*_{2}\right)\right\rangle \text { wf } \quad \forall k, \vdash\left\langle D\left(*_{1}\right), M_{k}\right\rangle \text { wf } \\
& \vdash\left\langle\left[\sum_{i=1}^{n} \ell_{i}: M_{i 1} \sum_{j=1}^{m} \ell_{j}: M_{j} *_{1}\right],\left[\sum_{i=1}^{n} \ell_{i}: M_{i 2} \sum_{k=1}^{o} \ell_{k}: M_{k} *_{2}\right]\right\rangle \mathbf{w f}
\end{aligned}
$$

$\vdash\langle M, M\rangle$ wf $\quad$ Well-formed Mappings

$\vdash\langle M, \varnothing\rangle$ wf

$$
\frac{\vdash\left\langle S_{1}, S_{2}\right\rangle \text { wf }}{\vdash\left\langle\left(S_{1}\right)_{R},\left(S_{2}\right)_{R}\right\rangle \text { wf }}
$$$$
\frac{S_{1} \sqsubseteq S_{3} \quad \vdash\left\langle S_{1}, S_{2}\right\rangle \text { wf }}{\vdash\left\langle\left(S_{3}\right)_{*},\left(S_{2}\right)_{O}\right\rangle \mathbf{w f}}
$$

Fig. 10. BRR $\mathrm{B}_{\varsigma}$ 's definition of well-formed evidence

see why, consider the set $\{[\ell: \operatorname{Int}] \mid \ell \in$ Label $\}$ : what is the most precise abstraction of this set when Label is an infinite set? The answer is that there is none, because this set features an infinite number of "optional" fields, and since bounded records only have finite fields, there is no best representative. The solution is to restrict the domain to hereditarily admit only collections of records that (1) have non-? bound for a finite set of field types; and (2) have either a finite set of potentially present fields (abstracts to a bounded record) or a finite set of absent fields (abstracts to a bounded row). The image of concretization satisfies these constraints, as do our operations on evidence. These finitary restrictions are analogous to the restrictions on the open sets of an infinite product topology [Munkres 2000].

Theorem 5.1. $\alpha$ and $\gamma$ for bounded records and rows form a Galois Connection.

Proof. Consequence of Soundness and Optimality Lemmas (see technical report [Bañados Schwerter et al. 2020]).

Well-formed Evidence. Using bounded records and rows, we develop a refined notion of evidence for $\mathrm{GTFL}_{\lessgtr}$. Fig. 10 defines the inductive structure of well-formed BRR evidence. Its structure is somewhat analogous to the original, but with richer distinctions. 


\subsection{Absent Labels Enable Sound Optimizations}

We motivate the introduction of bounded records and rows to solve associativity issues and to guarantee that programs with inconsistent ascriptions always fail. Associativity more broadly could also support program optimizations such as inlining and pre-composing evidence objects. Consider the following program:

\section{( $\lambda x:$ [?]. (if true then (if true then $x$ else $x::[l: \operatorname{lnt}, ?])$ else $x::[l:$ Bool,?]))}

This program relies on if branching to generate uses of consistent subtype join which will generate evidence objects that use the optional annotation. This program will produce an error when given as an argument any record that has an $l$ mapping. This is because the ascriptions impose inconsistent constraints: the function body must have type $\left[l:\right.$ Bool $_{O}$, ?] in the outermost if , and $\left[l: \operatorname{lnt}_{O}\right.$ ?] in the innermost if , thus $x$ must go through the composition of evidence on both types, reaching an inconsistency whenever the label is present.

While the sole introduction of optional fields would suffice to run this program properly, consider now an optimizing compiler that performs constant propagation in this function. The body of the function might then be optimized to $\lambda x . \varepsilon_{2} \varepsilon_{1} x$, with $\varepsilon_{2}=\left\langle\left[l: \mathrm{Bool}_{O}\right.\right.$, ?], [ $l: \mathrm{Bool}_{O}$, ?] $\rangle$ and $\varepsilon_{1}=\left\langle\left[l: \operatorname{Int}_{O}, ?\right],\left[l: \operatorname{Int}_{O}, ?\right]\right\rangle$. A more advanced optimizing compiler could try to perform this evidence composition ahead of time. Unfortunately, unless we introduce absent labels as in BRR, the only possible composition would be to coalesce the label $l$ into the row portion to generate the evidence pair $\langle[\mid ?],[\mid ?]\rangle$. This "optimization" changes the behaviour of our program, as arguments with a mapping for $l$ will now be accepted instead of producing an error. To achieve full associativity and soundness in the presence of optimizations like the above, we need absent labels.

\section{FORWARD COMPLETENESS AS A KEY TO ASSOCIATIVITY}

The previous section delves into the shortcomings of GTFL $_{\lesssim}$ 's evidence abstraction, diagnoses some evident information loss, and devises a new abstraction that retains the relevant information. Are these improvements sufficient? An example-driven approach can drive us closer to a solution, but ultimately we need more rigorous and comprehensive confirmation, which we now provide. Moreover, we do so by generalizing beyond $\mathrm{GTFL}_{\S}$, seeking sufficient criteria that can apply to future applications of AGT, regardless of the particulars of the type discipline or gradualization.

First, consider associativity. We must prove that $\varepsilon_{1} \stackrel{\circ}{(}\left(\varepsilon_{2} \stackrel{\circ}{9} \varepsilon_{3}\right)=\left(\varepsilon_{1} \stackrel{\circ}{9} \varepsilon_{2}\right) \stackrel{\circ}{9} \varepsilon_{3}$ holds for our new notion of evidence. A direct proof of this property is possible, but does not scale well. Each evidence object $\varepsilon$ is a pair of BRR types, and associativity must consider not only how they compose with one another, but also with the results of intermediate compositions. The case explosion is staggering.

Even after proving associativity, how does one confirm that the evidence abstraction is precise enough to protect type-based invariants? Is a second complex formal development necessary?

Fortunately no: there is another way. In the context of abstract model-checking, Giacobazzi and Quintarelli [2001] introduce the concept of forward completeness, ${ }^{15}$ which is dual to the concept of (backward) completeness that arises more naturally for abstract interpretation-based static analysis. The idea applies to any abstract function, but we present it here in domain-specific terms.

Definition 6.1 (Forward completeness). Let $\varepsilon$ denote evidence objects, ̊ denote consistent transitivity, and ; denote relational composition over fragments of static subtyping. Then $\circ$ is forward complete with respect to its evidence abstraction if $\gamma^{<:}\left(\varepsilon_{1}\right) ; \gamma^{<:}\left(\varepsilon_{2}\right)=\gamma^{<:}\left(\varepsilon_{1} \stackrel{\circ}{9} \varepsilon_{2}\right)$ for any two evidence objects $\varepsilon_{1}$ and $\varepsilon_{2}$.

${ }^{15}$ Sometimes called gamma-completeness or exactness 


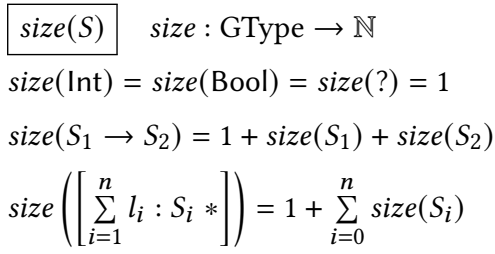

height $(S) \quad$ height $:$ GType $\rightarrow \mathbb{N}$
height $($ Int $)=$ height $($ Bool $)=\operatorname{height}(?)=1$
$\operatorname{height}\left(S_{1} \rightarrow S_{2}\right)=1+\max \left(\operatorname{height}\left(S_{1}\right)\right)\left(\operatorname{height}\left(S_{2}\right)\right)$
height $\left(\left[\sum_{i=1}^{n} l_{i}: S_{i} *\right]\right)=1+\max _{i=0}^{n} \operatorname{height}\left(S_{i}\right)$

Fig. 11. Size and height of a gradual type

Soundness of $\%$ with respect to relational composition ; implies $\gamma^{<:}\left(\varepsilon_{1}\right) ; \gamma^{<:}\left(\varepsilon_{2}\right) \subseteq \gamma^{<:}\left(\varepsilon_{1} \stackrel{\circ}{q} \varepsilon_{2}\right)$, which ensures that $\stackrel{\circ}{9}$, operating on abstract evidence objects, sufficiently overapproximates the behavior of ; on the meanings of those objects. Forward completeness implies that the reversecontainment also holds, which means that 9 exactly approximates ; for abstract objects. This means that $\%$ is a perfect stand-in for ; if we need only consider sets in the image of evidence concretization. In the case of AGT, such is exactly the case: evidence is initially introduced in terms of source gradual types, which are even less precise than evidence objects.

Lemma 6.2 (Relational Composition is closed wrt concretization). For any $\vdash \varepsilon_{1}$ wf and $\vdash \varepsilon_{2} \mathbf{w f}$, either $\gamma^{<:}\left(\varepsilon_{1}\right) ; \gamma^{<:}\left(\varepsilon_{2}\right)=\emptyset$, or there exists an $\vdash \varepsilon_{3}$ wf such that $\gamma^{<:}\left(\varepsilon_{1}\right) ; \gamma^{<:}\left(\varepsilon_{2}\right)=\gamma^{<:}\left(\varepsilon_{3}\right)$.

Proof. By double induction over $\vdash \varepsilon$ wf and $\vdash\langle M, M\rangle$ wf for $\varepsilon_{1}$ and $\varepsilon_{2}$.

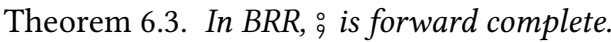

Proof. Consequence of Lemma 6.2.

It is straightforward to prove that forward completeness suffices for space-efficiency.

Theorem 6.4. If $\circ$ is forward-complete with respect to its evidence abstraction, then $\varepsilon_{1} \circ\left(\varepsilon_{2} \stackrel{\circ}{9} \varepsilon_{3}\right)=$ $\left(\varepsilon_{1} \stackrel{\circ}{9} \varepsilon_{2}\right) \stackrel{\circ}{9} \varepsilon_{3}$ for any evidence objects $\varepsilon_{1}, \varepsilon_{2}$ and $\varepsilon_{3}$.

Proof. A generalized Coq mechanization of this property can be found in Bañados Schwerter [2020].

The basic intuition is that relational composition is associative, so a forward-complete definition must also be. Of more practical interest, though, is that the statement of forward completeness involves only two evidence objects, compared to the three needed to state associativity. Thus, proving forward completeness requires far fewer cases than proving associativity directly. Additionally, forward completeness ensures that reasoning about transitivity of subtyping is as precise as operating directly with fragments of subtyping, as alluded to in Sec. 3. This precision matters most when considering precise enforcement of type invariants.

\section{BRR IS SPACE-EFFICIENT}

As the previous section proved that evidence composition is associative, we must only find a bound for evidence composition in the style of Herman et al. [2010] to guarantee that BRR is space efficient. Since an evidence object is just a pair of types, we can use the size of a gradual type as a proxy for the size of evidence. Fig. 11 defines structurally the size and the height of a gradual type. The size of a gradual type can be bound in terms of its height, and thus we can find a bound for the size of an evidence object in terms of the height of its components. 
Lemma 7.1 (Bound for size of Gradual Types in BRR). For any gradual type $S$,

$$
\operatorname{size}(S) \leq\left(3+\operatorname{dom}_{l}(S)\right)^{1+h e i g h t(S)}
$$

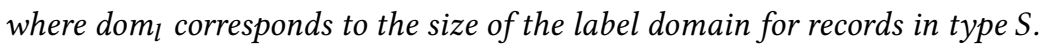

While this bound seems quite high, it is comparable to the bound established by Herman et al. [2010] for the size of coercions: Their structural types only include functions and references, so they only needed to consider binary tree branching at any level in their types. Once we introduce records, we must instead deal with arbitrarily-wide n-ary tree branching.

Definition 7.2 (Size and height of evidences in BRR).

$$
\begin{gathered}
\operatorname{size}(\varepsilon)=\operatorname{size}\left(\pi_{1}(\varepsilon)\right)+\operatorname{size}\left(\pi_{2}(\varepsilon)\right) \\
\operatorname{height}(\varepsilon)=\max \operatorname{height}\left(\pi_{1}(\varepsilon)\right) \operatorname{height}\left(\pi_{2}(\varepsilon)\right) \\
\operatorname{dom}_{l}(\varepsilon)=\max \operatorname{dom}_{l}\left(\pi_{1}(\varepsilon)\right) \operatorname{dom}_{l}\left(\pi_{2}(\varepsilon)\right)
\end{gathered}
$$

Since BRR is forward-complete, we can rely on Prop. 3.3 to reach a bound for evidence composition through a combination of bounds for gradual meet and initial evidence:

Lemma 7.3 (Height bounds in BRR). height $\left(S_{1} \sqcap S_{2}\right) \leq \max \left(\operatorname{height}\left(S_{1}\right)\right)\left(\operatorname{height}\left(S_{2}\right)\right)$;

$$
\begin{gathered}
\operatorname{height}\left(\mathcal{I}\left[\left[S_{1} \lesssim S_{2}\right]\right]\right) \leq \max \left(\operatorname{height}\left(S_{1}\right)\right)\left(\operatorname{height}\left(S_{2}\right)\right) ; \\
\operatorname{height}\left(\varepsilon_{1} \stackrel{\circ}{9} \varepsilon_{2}\right) \leq \max \left(\operatorname{height}\left(\varepsilon_{1}\right)\right)\left(\operatorname{height}\left(\varepsilon_{2}\right)\right) .
\end{gathered}
$$

We can use these lemmas to prove the following bound for evidence composition:

Theorem 7.4 (Bound for the size of evidence composition).

$$
\operatorname{size}\left(\varepsilon_{1} \stackrel{\circ}{9} \varepsilon_{2}\right) \leq 2 *\left(3+\max \left(\operatorname{dom}_{l}\left(\varepsilon_{1}\right)\right)\left(\operatorname{dom}_{l}\left(\varepsilon_{2}\right)\right)\right)^{1+\max \left(h e i g h t\left(\varepsilon_{1}\right)\right)\left(\operatorname{height}\left(\varepsilon_{2}\right)\right)}
$$

Proof. A Coq proof for Lemma 7.3 and Theorem 7.4 can be found in Bañados Schwerter [2020].

Since any evidence composition is bound by the height and label domain of its components, we can establish a bound for any evidence composition happening in a program by collecting the greatest height and domain, respectively:

Theorem 7.5 (Bound for evidence compositions in a program). Let $\mathbf{e}$ be a program. There exists an $\varepsilon_{h}$ appearing in a subterm of $\mathbf{e}$, such that for any evidences $\varepsilon_{1}$ and $\varepsilon_{2}$ appearing in any subterms of $\mathbf{e}$,

$$
\operatorname{size}\left(\varepsilon_{1} \stackrel{\circ}{\varepsilon_{2}}\right) \leq 2 *\left(3+\operatorname{dom}_{l}(\mathbf{e})\right)^{1+h e i g h t\left(\varepsilon_{h}\right)}
$$

where $\operatorname{dom}_{l}(\mathbf{e})$ denotes the domain of labels for evidence appearing in subterms of $\mathbf{e}$.

We can use this bound as a proof of Prop. 4.5. Combining this with Theorems 6.3 and 6.4 as a proof of Prop. 4.4, thus we have a space efficient semantics.

\section{DISCUSSION}

As part of their analysis of counter-example guided abstraction refinement, Giacobazzi and Quintarelli [2001] develop a constructive approach to systematically refine abstractions to be complete with respect to an operation. Though we developed BRR manually, future AGT-based designs could employ their techniques to more systematically produce a forward complete evidence abstraction starting from the default proposed by Garcia et al. [2016].

Space efficiency is but one operational criteria of gradually-typed languages. In future work we will develop generalized techniques for reducing the density of evidence objects in programs. Using AGT off-the-shelf leads to a semantics that saturates even precisely typed code with evidence. We 
believe that the refined notions of evidence in this work may help with understanding how to minimize the amount of runtime checks in programs.

As has been observed by others [Toro et al. 2018, 2019], the guarantees provided automatically by AGT are not sufficient to yield a satisfactory gradually typed language. Strong type-based reasoning may necessitate additional tuning and proof obligations. The two works cited above demonstrate this in the context of relational type invariants like parametricity and information-flow control. Furthermore, the evidence composition developed by Toro et al. [2018] is associative, but this alone did not suffice to achieve their noninterference result. This is the first work to demonstrate that an AGT-based gradual type system that enforces non-relational type invariants can need additional tuning. However, in the non-relational case, forward-completeness suffices. Nonetheless, these experiences suggest a desire for additional properties beyond those proposed by Siek et al. [2015] It is worth noting that blame, another facet of gradual typing, does not appear to help here: blame theorems do not indicate which programs should fail, only which parties may or may not be responsible when they do.

In this paper we have grounded our exposition in the concrete example of Bounded Rows and Records (BRR). While forward-completeness guarantees associativity of composition, and thus suffices for an observationally equivalent semantics that is bound for stack, it does not guarantee space-efficiency on its own, as one must still prove that evidence composition is itself bounded. In the particular case of BRR, this last proof is supported by the fact that the underlying static subtyping is a transitive and syntax-directed relation. Our work should transfer to other typing disciplines with these constraints, and provides more precise guidelines for future AGT developments to systematically provide space-efficiency. To the best of our knowledge, we are the first to prove space efficiency in a gradual language with records (and record subtyping).

\section{CONCLUSION}

Abstracting gradual typing provides a powerful scaffold, based in the theory of abstract interpretation, that helps designers construct gradually typed variants of static type disciplines. This paper draws on additional concepts from abstract interpretation to inform and improve those designs. In doing so, we provide a deeper analysis of the concept of evidence in AGT, and devise a general, though not necessarily universally feasible, tool for constructing space-efficient semantics that precisely enforce the invariants implied by source gradual types. We propose that, regardless of the source gradual type abstraction, a forward-complete runtime abstraction should be the new default when possible. Space-efficiency, and precise runtime semantics are sufficient motivation.

\section{ACKNOWLEDGMENTS}

We acknowledge the support of the Natural Sciences and Engineering Research Council of Canada (NSERC). Cette recherche a été financée par le Conseil de recherches en sciences naturelles et en génie du Canada (CRSNG). The authors also thank Phil Wadler, Yuchong Pan, Peter Thiemann, and the anonymous reviewers.

\section{REFERENCES}

Amal Ahmed, Dustin Jamner, Jeremy G. Siek, and Philip Wadler. 2017. Theorems for Free for Free: Parametricity, with and without Types. Proc. ACM Program. Lang. 1, ICFP, Article 39 (Aug. 2017), 28 pages. https://doi.org/10.1145/3110283

Felipe Bañados Schwerter. 2020. Coquihalla/agt-moving-forward: Supporting Artifacts from POPL '21 submission. https: //doi.org/10.5281/zenodo.4111019

Felipe Bañados Schwerter, Alison M. Clark, Khurram A. Jafery, and Ronald Garcia. 2020. Abstracting Gradual Typing Moving Forward: Precise and Space-Efficient (Technical Report). arXiv:2010.14094 [cs.PL]

Felipe Bañados Schwerter, Ronald Garcia, and Éric Tanter. 2014. A Theory of Gradual Effect Systems. In 19th ACM SIGPLAN Conference on Functional Programming (ICFP 2014). ACM Press, Gothenburg, Sweden, 283-295. https://doi.org/10.1145/ 
2628136.2628149

Patrick Cousot and Radhia Cousot. 1977. Abstract interpretation: A unified lattice model for static analysis of programs by construction or approximation of fixpoints. In POPL. ACM Press, Los Angeles, CA, USA, 238-252. https://doi.org/10. $1145 / 512950.512973$

Tim Disney and Cormac Flanagan. 2011. Gradual information flow typing. https://users.soe.ucsc.edu/ cormac/papers/ stop11.pdf

Ronald Garcia and Matteo Cimini. 2015. Principal Type Schemes for Gradual Programs. In 42nd ACM SIGPLAN-SIGACT Symposium on Principles of Programming Languages (POPL 2015). ACM Press, New York, NY, USA, 303-315. https: //doi.org/10.1145/2676726.2676992

Ronald Garcia, Alison M. Clark, and Éric Tanter. 2016. Abstracting Gradual Typing. In 43rd ACM SIGPLAN-SIGACT Symposium on Principles of Programming Languages (St Petersburg, FL, USA). ACM Press, New York, NY, USA, 429-442. https://doi.org/10.1145/2837614.2837670

Roberto Giacobazzi and Elisa Quintarelli. 2001. Incompleteness, Counterexamples, and Refinements in Abstract ModelChecking. In Proceedings of the 8th International Symposium on Static Analysis (SAS '01). Springer-Verlag, London, UK, UK, 356-373. http://dl.acm.org/citation.cfm?id=647170.718288

Fritz Henglein. 1994. Dynamic typing: syntax and proof theory. Science of Computer Programming 22, 3 (June 1994), 197-230. https://doi.org/10.1016/0167-6423(94)00004-2

David Herman, Aaron Tomb, and Cormac Flanagan. 2010. Space-efficient Gradual Typing. Higher Order Symbol. Comput. 23, 2 (June 2010), 167-189. https://doi.org/10.1007/s10990-011-9066-z

Lintaro Ina and Atsushi Igarashi. 2011. Gradual typing for generics. In 26th ACM SIGPLAN Conference on Object-Oriented Programming Systems, Languages and Applications (OOPSLA 2011). ACM Press, New York, NY, USA, 609-624. https: //doi.org/10.1145/2048066.2048114

James R. Munkres. 2000. Topology: Second Edition. Prentice Hall, Upper Saddle River, NJ.

Max S. New, Dustin Jamner, and Amal Ahmed. 2019. Graduality and Parametricity: Together Again for the First Time. Proc. ACM Program. Lang. 4, POPL, Article 46 (Dec. 2019), 32 pages. https://doi.org/10.1145/3371114

Benjamin C. Pierce. 2002. Types and programming languages. MIT Press, Cambridge, MA, USA.

D. Rémy. 1989. Type Checking Records and Variants in a Natural Extension of ML. In POPL (Austin, Texas, USA). ACM, New York, NY, USA, 77-88. https://doi.org/10.1145/75277.75284

Davide Sangiorgi. 2011. Introduction to Bisimulation and Coinduction. Cambridge University Press, New York, NY, USA.

Ilya Sergey and Dave Clarke. 2012. Gradual Ownership Types. In Proc. European Symposium on Programming Languages and Systems (ESOP '12, Vol. 7211), Helmut Seidl (Ed.). Springer-Verlag, Tallinn, Estonia, 579-599. https://doi.org/10.1007/9783-642-28869-2_29

Jeremy Siek and Walid Taha. 2007. Gradual typing for objects. In Proc. European Conference on Object-oriented Programming (ECOOP '07, 4609), Erik Ernst (Ed.). Springer-Verlag, Berlin, Germany, 2-27. https://doi.org/10.1007/978-3-540-73589-2_2

Jeremy Siek and Philip Wadler. 2010. Threesomes, with and without blame. In Proc. Symposium on Principles of Programming Languages (Madrid, Spain) (POPL '10). ACM, New York, NY, USA, 365-376. https://doi.org/10.1145/1570506.1570511

Jeremy G. Siek and Walid Taha. 2006. Gradual Typing for Functional Languages. In Proc. Scheme and Functional Programming Workshop. University of Chicago Technical Report, Chicago, IL, USA, 81-92. http://scheme2006.cs.uchicago.edu/ scheme2006.pdf

Jeremy G. Siek, Michael M. Vitousek, Matteo Cimini, and John Tang Boyland. 2015. Refined Criteria for Gradual Typing. In SNAPL. Schloss Dagstuhl, Asilomar, California, USA, 274-293. https://doi.org/10.4230/LIPIcs.SNAPL.2015.274

Sam Tobin-Hochstadt and Matthias Felleisen. 2006. Interlanguage Migration: From Scripts to Programs. In OOPSLA. ACM, New York, NY, USA, 964-974. https://doi.org/10.1145/1176617.1176755

Matías Toro, Ronald Garcia, and Éric Tanter. 2018. Type-Driven Gradual Security with References. ACM Transactions on Programming Languages and Systems 40, 4, Article 16 (Dec. 2018), 55 pages. https://doi.org/10.1145/3229061

Matías Toro, Elizabeth Labrada, and Éric Tanter. 2019. Gradual Parametricity, Revisited. Proc. ACM Program. Lang. 3, POPL, Article 17 (Jan. 2019), 30 pages. https://doi.org/10.1145/3290330

Matías Toro and Éric Tanter. 2020. Abstracting gradual references. Science of Computer Programming 197 (2020), 102496. https://doi.org/10.1016/j.scico.2020.102496

Preston Tunnell Wilson, Ben Greenman, Justin Pombrio, and Shriram Krishnamurthi. 2018. The Behavior of Gradual Types: A User Study. In DLS (Boston, MA, USA). ACM, New York, NY, USA, 1-12. https://doi.org/10.1145/3276945.3276947

Philip Wadler and Robert Bruce Findler. 2009. Well-Typed Programs Can't Be Blamed. In Proc. European Symposium on Programming Languages (York, UK) (ESOP '09). Springer-Verlag, Berlin, 1-16. https://doi.org/10.1007/978-3-642-00590-9_1

Andrew K. Wright and Matthias Felleisen. 1994. A Syntactic Approach to Type Soundness. Information and Computation 115, 1 (Nov. 1994), 38-94. https://doi.org/10.1006/inco.1994.1093 